\title{
Designing Broadband over Power Lines Networks Using the Techno-Economic Pedagogical (TEP) Method - Part I: Overhead High Voltage Networks and Their Capacity Characteristics
}

\author{
Athanasios G. Lazaropoulos ${ }^{1,2, *}$ \\ 1: School of Electrical and Computer Engineering, National Technical University of Athens (NTUA), \\ 9 Iroon Polytechniou Street, Zografou, Athens, Greece 15780 \\ 2: Department of Electrical Engineering Educators, School of Pedagogical and Technological Education \\ (ASPETE), Station Eirini HSAP, Heraklio Attikis, Athens, Greece 14121
}

Received February 8, 2015; Accepted March 2, 2015; Published March 8, 2015

\begin{abstract}
This pair of papers proposes the techno-economic pedagogical (TEP) method that is suitable for designing Broadband over Power Lines (BPL) networks in transmission and distribution power grids. During the presentation of TEP method, a review of the recent research efforts concerning BPL networks across transmission and distribution power grids is given.

In this first paper, TEP method demonstrates to undergraduate electrical and computer engineering (ECE) students the interaction between two apparently irrelevant fields of their ECE program: Microwave Engineering and Engineering Economics. On the basis of a set of linear simplifications and suitable techno-economic metrics concerning transmission and capacity properties of overhead High Voltage Broadband over Power Lines (HV/BPL) networks, TEP method reveals the broadband potential of overhead HV/BPL networks to ECE students when different overhead HV/BPL topologies, electromagnetic interference $(\mathrm{EMI})$ regulations and noise conditions are considered.
\end{abstract}

Keywords: Education; Educational Policy; Comparative Education; Faculty of Electrical and Computer Engineering; Microwave Engineering; Engineering Economics; Broadband over Power Lines (BPL) modeling; Power Line Communications (PLC); overhead High-Voltage (HV) power lines; Capacity

\section{Nomenclature and Abbreviations}

\begin{tabular}{ll|ll}
\hline Nomenclature & Abbr. & Nomenclature & Abbr. \\
\hline Smart Grid & SG & Differential Modes & DMs \\
Electrical and Computer Engineering & ECE & Wire-to-Wire & WtW \\
Broadband over Power Lines & BPL & Wire-to-Ground & WtG \\
High-Voltage & HV & Electromagnetic Compatibility & EMC \\
Techno-Economic Pedagogical & TEP & Injected Power Spectral Density & IPSD \\
Multiconductor Transmission Line & MTL & Additive White Gaussian Noise & AWGN \\
Electromagnetic Interference & EMI & Multiple-Input Multiple-Output & MIMO \\
Transmission Line & TL & Supervisory Control and Data Acquisition & SCADA \\
Common Mode & CM & & \\
\hline
\end{tabular}

\section{Introduction}

Modern microwave engineering refers to the study and design of microwave circuits, components and systems as well as the characterization of corresponding 
phenomena like noise, nonlinear effects, etc. Fundamental principles of electromagnetics, such as Maxwell's equations, wave propagation, network analysis and other design principles, are applied to analysis, design and measurement techniques in this field [1]. In fact, a plethora of microwave engineering applications emphasizes the widespread use of microwave technology for communications systems, wireless local area networks and many other systems related to the information infrastructure and the SG transition [2].

Except for the understanding of the fundamentals of microwave engineering, undergraduate ECE students should consider the economic impact and viability of each potential application along with its technical aspects. Engineering economics is the required subset of the economics that is applied to engineering projects [3]. To promote the interaction between these two fields of the ECE program -i.e., Microwave Engineering and Engineering Economics-, the teaching method of case studies, which has been widely applied in relevant engineering, economic and management fields, is also applied in this paper [4]-[7].

Based on the case study of the deployment of overhead BPL networks across the overhead transmission -i.e., HV- and distribution power grids, the new TEP method that is suitable for designing these overhead BPL networks is proposed. TEP method maintains its simplicity in understanding from ECE students without, however, losing its conceptual contact with the microwave engineering phenomena that characterize the propagation and transmission characteristics in overhead BPL networks. In this paper, TEP method focuses on the design of overhead HV/BPL networks [8]-[11].

TEP method combines the well-verified hybrid method, which is usually employed to examine the behavior of BPL transmission channels installed on MTL structures [8]-[17], with a set of linear simplifications, which concerns the operation of overhead HV/BPL networks. More specifically, the hybrid method, which is a careful cascade of well-known microwave engineering techniques, comprises: (i) the bottom-up approach: It combines MTL theory with similarity transformations determining the excited modes of overhead HV MTL configurations in terms of their propagation constants; and (ii) the top-down approach: It consists of the concatenation of multidimensional $T$-matrices of network modules (TM2 method) having as outputs some important techno-economic metrics of overhead HV/BPL networks such as end-to-end channel attenuation and capacity. As it is verified in this paper, the set of linear simplifications transforms the complicated hybrid method into a straightforward process without seriously affecting the validity and the accuracy of the used techno-economic metrics.

On the basis of the aforementioned two techno-economic metrics, the diverse nature of overhead HV/BPL networks is reviewed and highlighted in the $3-30 \mathrm{MHz}$ frequency band [10], [12]-[16]. The main contribution of TEP method is that permits to ECE students to clearly and intuitively understand the impact of several factors such as the length of overhead HV/BPL networks, the imposed power constraints in order to comply with EMI regulations concerning BPL emissions and the noise conditions. Finally, the simplicity of TEP method allows its easy implementation in computers, the further experimentation by ECE students and the familiarization of ECE students with the fundamentals of engineering economics such as the trade-off relations among involved BPL system parameters.

The rest of this paper is organized as follows: In Section II, the overhead HV configuration adopted in this paper is illustrated. Section III synopsizes the main features of overhead HV/BPL transmission. Section IV reviews the EMI regulations, the noise 
characteristics and the evaluation of the capacity delivered by overhead HV/BPL networks. In Section V, numerical results and conclusions are provided, aiming at revealing the interaction of Microwave Engineering with Engineering Economics. Section VI concludes this paper.

\section{A Brief Overview of Overhead HV MTL Configurations}

Overhead HV MTL configurations are mainly classified in the electrical power industry by: (i) their voltage level; (ii) their number of MTL circuits per each tower; and (iii) the number of neutral conductors per each tower [8]-[11].

A typical case of $150 \mathrm{kV}$ single-circuit overhead HV MTL configuration is depicted in Fig. 1. Three parallel phase conductors spaced by $\Delta_{\mathrm{p}}^{150 \mathrm{kV}}$ are suspended at heights $h_{\mathrm{p}}^{150 \mathrm{kV}}$ above lossy ground -conductors 1, 2 and 3-. Moreover, two parallel neutral conductors spaced by $\Delta_{\mathrm{n}}^{150 \mathrm{kV}}$ hang at heights $h_{\mathrm{n}}^{150 \mathrm{kV}}$-conductors 4 and 5(for more details see in [8], [11]). This three-phase five-conductor $(n=5)$ overhead HV MTL configuration is considered in the present work consisting of ACSR GROSBEK conductors [10], [11], [18], [19].

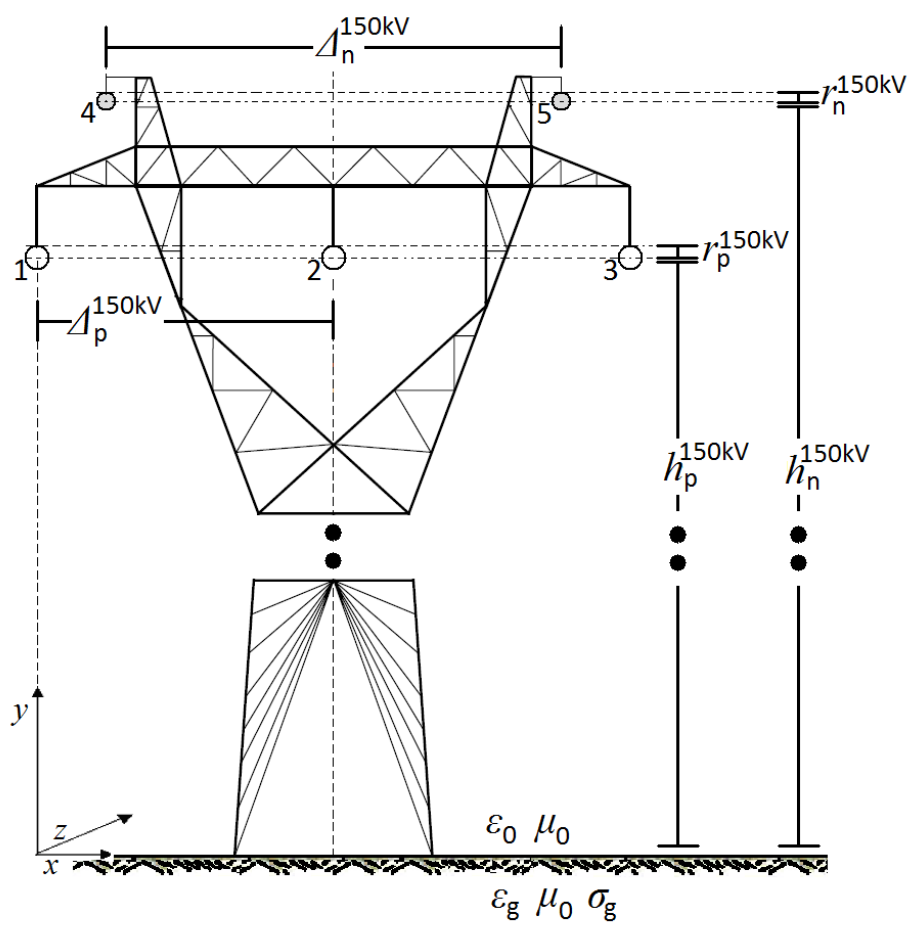

Figure 1. 150kV single-circuit overhead HV MTL structure [8], [10], [11], [18], [19].

The impact of imperfect ground on signal propagation via overhead $\mathrm{HV} / \mathrm{BPL}$ networks was analyzed in [8]-[16], [20]-[23]. In accordance with these analyses, the ground is considered as the reference conductor. Its conductivity $\sigma_{g}$ is assumed equal to 
$5 \mathrm{mS} / \mathrm{m}$ while its relative permittivity $\varepsilon_{r g}$ is assumed equal to 13 , which is a realistic scenario [8]-[11], [13], [15], [20].

\section{The Modal Analysis of Overhead HV/BPL Networks through the Prism of Hybrid and TEP Method}

According to microwave engineering, the standard TL theory bridges the gap between field analysis and basic circuit theory when the phenomenon of wave propagation on TLs is examined [2]. Already analyzed in [8]-[16], through a matrix approach, the standard TL analysis can be treated as a subcase of the MTL case that involves more than two conductors. Compared to a two-conductor line supporting one forward- and one backward-traveling wave, an overhead HV MTL structure with $n$ conductors parallel to the $z$ axis as depicted in Fig 1 may support $n$ pairs of forwardand backward-traveling waves with corresponding propagation constants. Each pair of forward- and backward-traveling waves is referred to as a mode.

\section{A. Modal Propagation Constants}

The $n$ modes that are supported by the overhead HV MTL configuration of Fig. 1

are: (i) the $\mathrm{CM}$ where $\gamma_{\mathrm{CM}}$ constitutes its propagation constant; and (ii) the $n-1 \mathrm{DMs}\left(\mathrm{DM}_{i-1}, i=2, \ldots, n\right)$ where $\gamma_{\mathrm{DM} i-1}, i=2, \ldots, n$ constitute the propagation constants of $\mathrm{DM}_{i-1}, i=2, \ldots, n$, respectively. The spectral behavior of the modal propagation constants has been thoroughly investigated in [8]-[11]. In Fig. 2(a), the attenuation coefficients $\alpha_{\mathrm{CM}}=\operatorname{Re}\left\{\gamma_{\mathrm{CM}}\right\}$ and $\alpha_{\mathrm{DM} i-1}=\operatorname{Re}\left\{\gamma_{\mathrm{DM} i-1}\right\}, i=2, \ldots, n$ of the $\mathrm{CM}$ and the $n-1 \mathrm{DMs}$, respectively, which are evaluated using the hybrid method, are plotted versus frequency for the overhead HV MTL configuration depicted in Fig. 1. In Fig. 2(c), the phase delays $\beta_{\mathrm{CM}}=\operatorname{Im}\left\{\gamma_{\mathrm{CM}}\right\}$ and $\beta_{\mathrm{DM} i-1}=\operatorname{Im}\left\{\gamma_{\mathrm{DM} i-1}\right\}, i=2, \ldots, n$ of the $\mathrm{CM}$ and the $n-1$ DMs, respectively, which are evaluated using the hybrid method, are plotted versus frequency for the same configuration. Note that $\operatorname{Re}\{\{\}$ and $\operatorname{Im}\{\cdot\}$ returns the real and the imaginary part of a complex number, respectively.

To bypass the complicated propagation analysis of the bottom-up approach of the hybrid method and to increase the ECE students' intuitiveness of the following techno-economic analysis, TEP method proposes that the attenuation coefficients and the phase delays of the $\mathrm{CM}$ and the DMs can be replaced by their respective linear approximations with satisfactory accuracy. Therefore, the mean values of the attenuation coefficients of the CM and the $n-1$ DMs are plotted versus frequency in Fig. 2(b) while the results of applying the linear regression to the phase delays of the same modes are plotted versus frequency in Fig 2(d).

\section{B. Modal Transfer Functions}

As it has already been presented in [8]-[16], TM2 method that is subcomponent of the top-down approach of the hybrid method, which is based on the scattering matrix theory of microwave engineering and detailed in [8], models the modal spectral behavior between $V_{i}^{\mathrm{m}}(z), i=1, \ldots, n$ and $V_{j}^{\mathrm{m}}(0), j=1, \ldots, n$ proposing operators $H_{i, j}^{\mathrm{m}}\{\cdot\}, i, j=1, \ldots, n$ so that 


$$
\mathbf{V}^{\mathrm{m}}(z)=\mathbf{H}^{\mathrm{m}}\left\{\mathbf{V}^{\mathrm{m}}(0)\right\}
$$

where $\mathbf{V}^{\mathrm{m}}(z)=\left[\begin{array}{llll}V_{1}^{\mathrm{m}}(z) & \cdots & V_{n}^{\mathrm{m}}(z)\end{array}\right]^{\mathrm{T}}$ are the modal voltages for the given overhead $\mathrm{HV}$ MTL configuration corresponding to the aforementioned $n$ supported modes, $[\cdot]^{\mathrm{T}}$ denotes the transpose of a matrix, $\mathbf{H}^{\mathrm{m}}\{\}$ is the $n \times n$ modal transfer function matrix whose elements $H_{i, j}^{\mathrm{m}}\{\}, i, j=1, \ldots, n$ with $i=j$ are the modal co-channel transfer functions, while those $H_{i, j}^{\mathrm{m}}\{\{\}, i, j=1, \ldots, n$ with $i \neq j$ are the modal cross-channel transfer functions and $H_{i, j}^{\mathrm{m}}$ denotes the element of matrix $\mathbf{H}^{\mathrm{m}}\{\cdot\}$ in row $i$ of column $j$. A strong aspect of TEP method is that achieves to totally bypass the top-down approach by appropriately scaling modal propagation constants since it focuses only on overhead HV/BPL topologies without branches (see also in Section V).

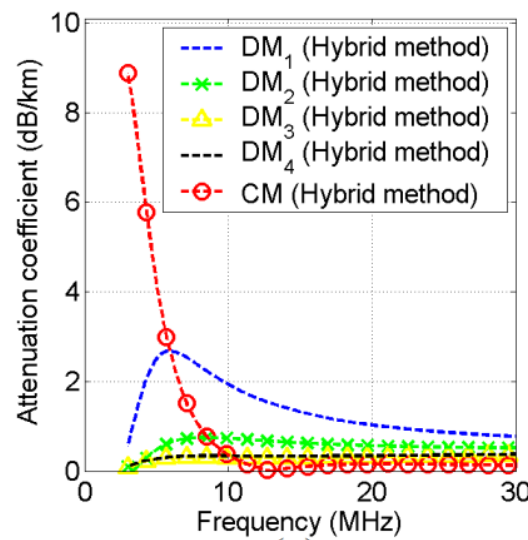

(a)

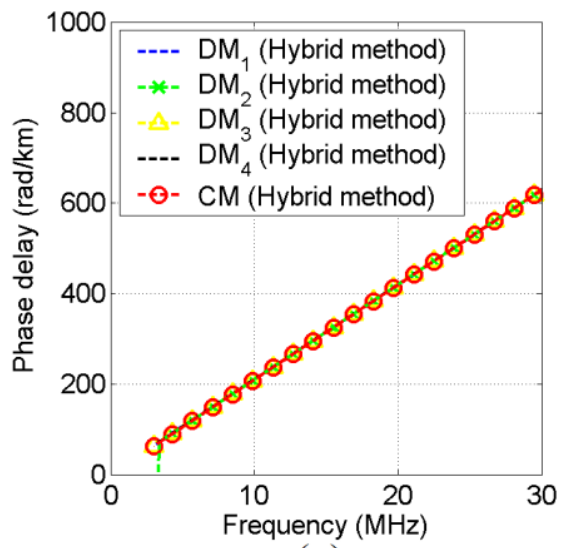

(c)

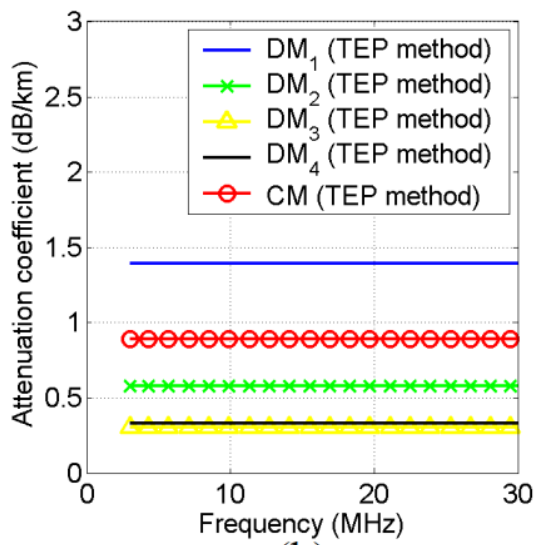

(b)

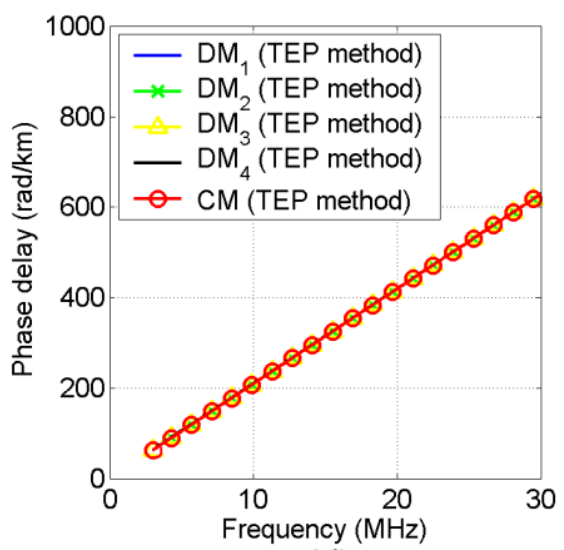

(d)

Figure 2. Frequency spectra of an $150 \mathrm{kV}$ single-circuit overhead HV multiconductor structure when different methods are applied in the $3-30 \mathrm{MHz}$ frequency band (the subchannel frequency spacing is equal to $0.1 \mathrm{MHz}$ ). (a, b) Attenuation coefficients. (c, d) Phase delays.

\section{Transfer Functions and Coupling Matrices}


Based on the modal analysis of Section IIIB and eq. (1), the $n \times n$ transfer function matrix $\mathbf{H}\{\cdot\}$ relating $\mathbf{V}(z)$ with $\mathbf{V}(0)$ through

$$
\mathbf{V}(z)=\mathbf{H}\{\mathbf{V}(0)\}
$$

is determined from

$$
\mathbf{H}\{\cdot\}=\mathbf{T}_{\mathrm{V}} \cdot \mathbf{H}^{\mathrm{m}}\{\cdot\} \cdot\left[\mathbf{T}_{\mathrm{V}}\right]^{-1}
$$

where $\mathbf{V}(z)=\left[\begin{array}{llll}V_{1}(z) & \cdots & V_{n}(z)\end{array}\right]^{\mathrm{T}}$ are the line voltages for the given overhead HV MTL configuration and $\mathbf{T}_{\mathrm{V}}$ is $n \times n$ matrix depending on the overhead HV MTL configuration, the frequency and the physical properties of the cables [8]-[16].

According to how signals are injected onto overhead HV/BPL TLs, two different coupling schemes exist, namely: (i) WtW coupling schemes, when the signal is injected between two conductors. This type of coupling scheme is outside of the scope of the TEP method; and (ii) WtG coupling schemes. When the signal is injected onto one conductor and returns via the ground; say between conductor $s, s=1, \ldots, n$ and the ground. Then, the coupling $\mathrm{WtG}$ transfer function $H^{\mathrm{WtG}}\{\cdot\}$ is given from [8]-[12]

$$
H^{\mathrm{WtG}}\{\cdot\}=\mathbf{A}^{\mathrm{WtG}} \cdot \mathbf{H}^{\mathrm{m}}\{\cdot\} \cdot \mathbf{B}^{\mathrm{WtG}}
$$

where

$$
\begin{aligned}
& \mathbf{A}^{\mathrm{WtG}}=\left[\mathbf{C}^{\mathrm{WtG}}\right]^{\mathrm{T}} \cdot \mathbf{T}_{\mathrm{V}} \\
& \mathbf{B}^{\mathrm{WtG}}=\left[\mathbf{T}_{\mathrm{V}}\right]^{-1} \cdot \mathbf{C}^{\mathrm{WtG}}
\end{aligned}
$$

are the coupling matrices related to the applied coupling scheme and overhead HV MTL configuration and $\mathbf{C}^{\mathrm{WtG}}$ is the $n \times 1 \mathrm{WtG}$ coupling column vector with zero elements except in the row $s$ where the value is equal to 1 [24]. $\mathrm{WtG}$ coupling between conductor $s$ and ground will be denoted as $\mathrm{WtG}^{s}$, hereafter.

Actually, $\quad \mathbf{A}^{\mathrm{WtG}}=\left[\begin{array}{lll}A_{1}^{\mathrm{WtG}} & \cdots & A_{n}^{\mathrm{WtG}}\end{array}\right] \quad$ and $\quad \mathbf{B}^{\mathrm{WtG}}=\left[\begin{array}{llll}B_{1}^{\mathrm{WtG}} & \cdots & B_{n}^{\mathrm{WtG}}\end{array}\right]^{\mathrm{T}} \quad$ are $1 \times n$ coupling row vector and $n \times 1 \mathrm{WtG}$ coupling column vector, respectively, describing the contribution of each modal transfer function to the WtG coupling scheme one. The absolute value of real and the imaginary part of the elements of coupling matrix $\mathbf{A}^{\mathrm{WtG}}$ are plotted versus frequency in Figs. 3(a) and 3(b), respectively, when $\mathrm{WtG}^{1}$ coupling scheme is applied and the hybrid method is adopted. In Figs. 3(c)-(i), the same plots are given in the case of $\mathrm{WtG}^{2}, \mathrm{WtG}^{3}, \mathrm{WtG}^{4}$ and $\mathrm{WtG}^{5}$ coupling schemes, respectively.

Similarly to the modal propagation constants, in order to completely bypass the application of the hybrid method and to simplify the following techno-economic analysis to ECE students, TEP method argues that the real parts of the elements of coupling matrix $\mathbf{A}^{\mathrm{WtG}}$ can be replaced by their mean values while their imaginary parts can be assumed equal to 0 in the frequency range of BPL operation. Hence, in Figs. 3(a)-(i), the same curves are plotted when TEP method is adopted. Same approximations are assumed in the case of the real and imaginary parts of the elements of the coupling matrix $\mathbf{B}^{\mathrm{WtG}}$.

From Figs. 2(a)-(d) and 3(a)-(j), it is evident that TEP method achieves to straightforward and efficiently describe all the propagation parameters involved in the determination of coupling $\mathrm{WtG}$ transfer functions of eq. (4). Actually, the propagation parameters of TEP method can be available $a b$ initio for given overhead HV MTL 
configuration and $\mathrm{WtG}$ coupling scheme. However, the overall performance of TEP method needs to be verified against other well-validated results, such as those derived from the hybrid method, for different techno-economic metrics (see in Section V).

\section{The Capacity Analysis of Overhead HV/BPL Networks Using the Hybrid and TEP Method}

Based on suitable techno-economic metrics, such as end-to-end channel attenuation and capacity of this paper, ECE students can assess the performance of overhead HV/BPL networks through the TEP method and, at the same time, TEP method can facilitate the design of overhead HV/BPL networks. More specifically, as it concerns the end-to-end channel attenuation, the propagation analysis of Section III is sufficient.

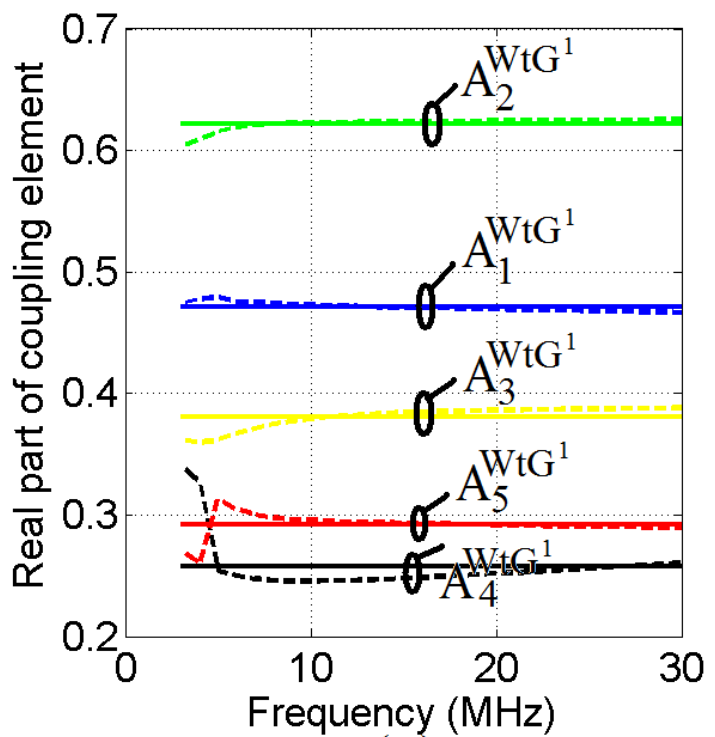

(a)

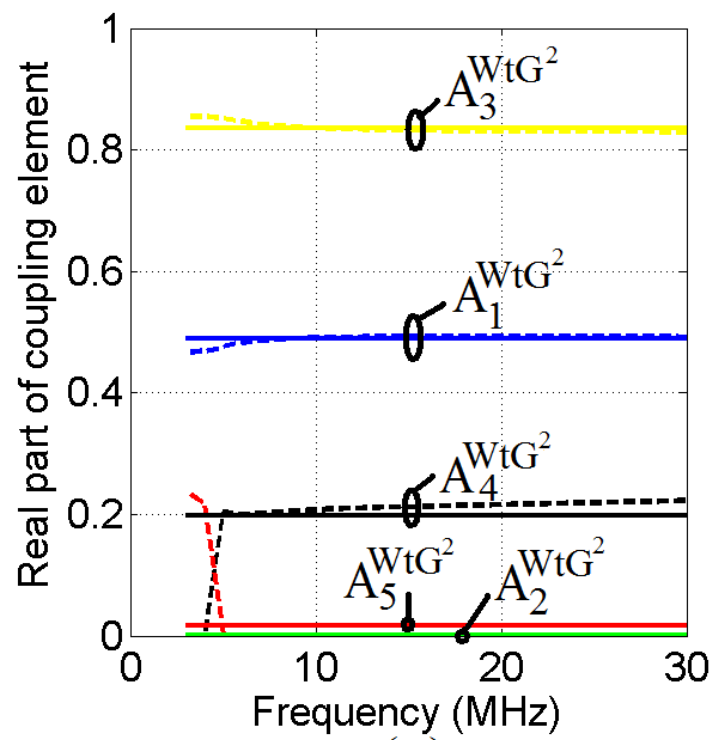

(c)

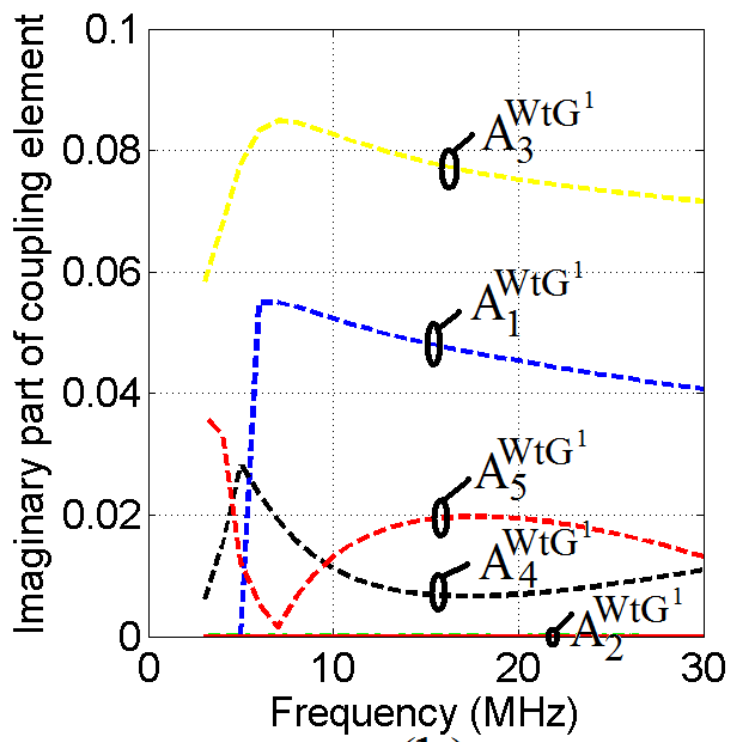

(b)

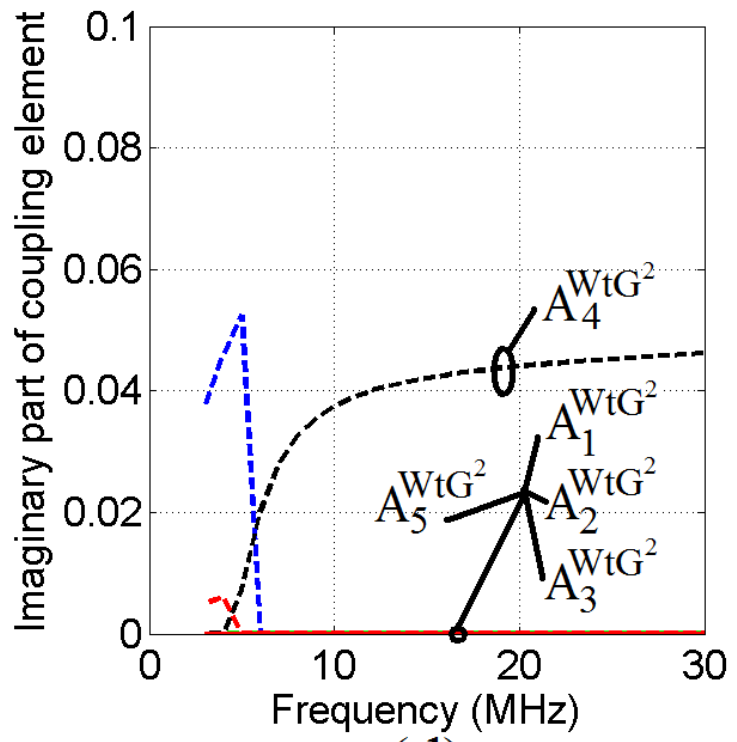

(d) 

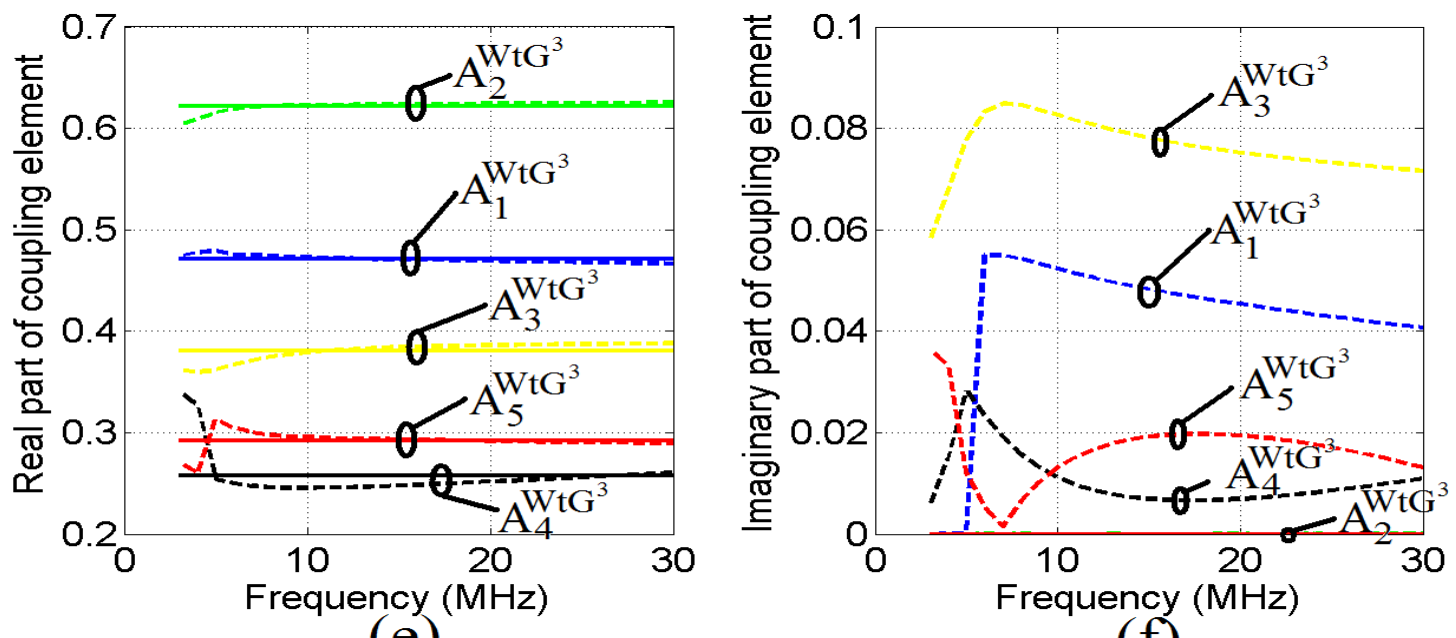

(e)

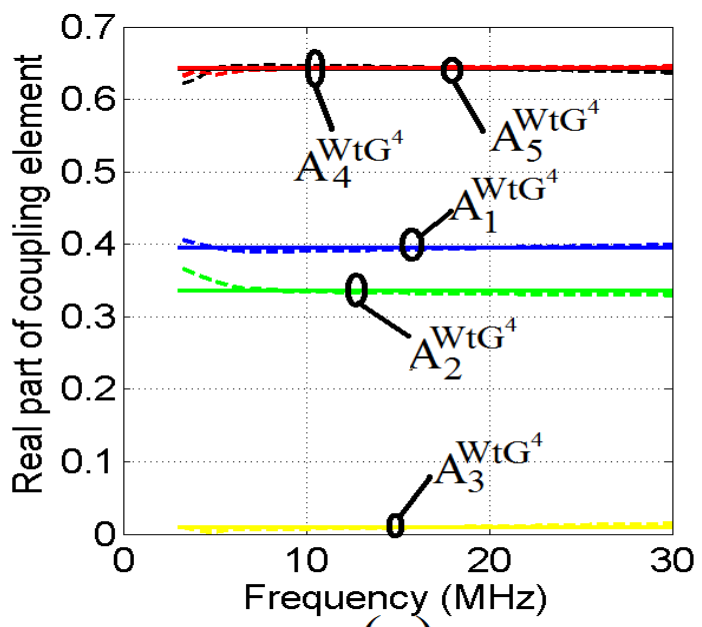

(g)

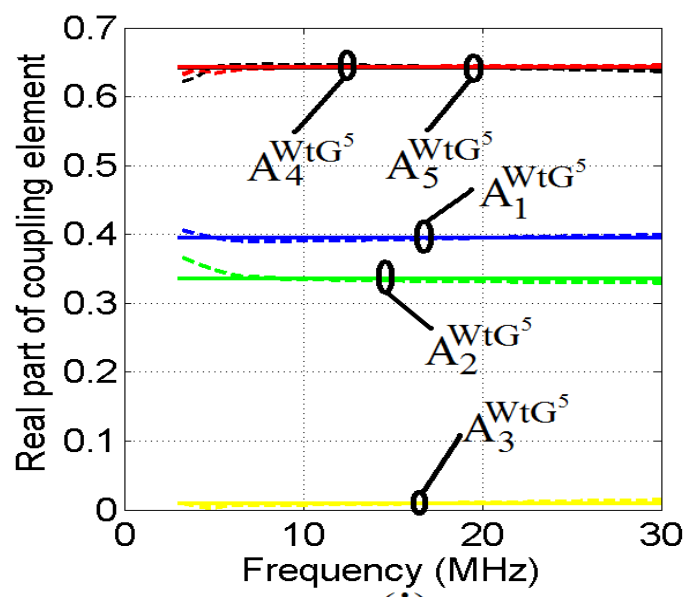

(i)

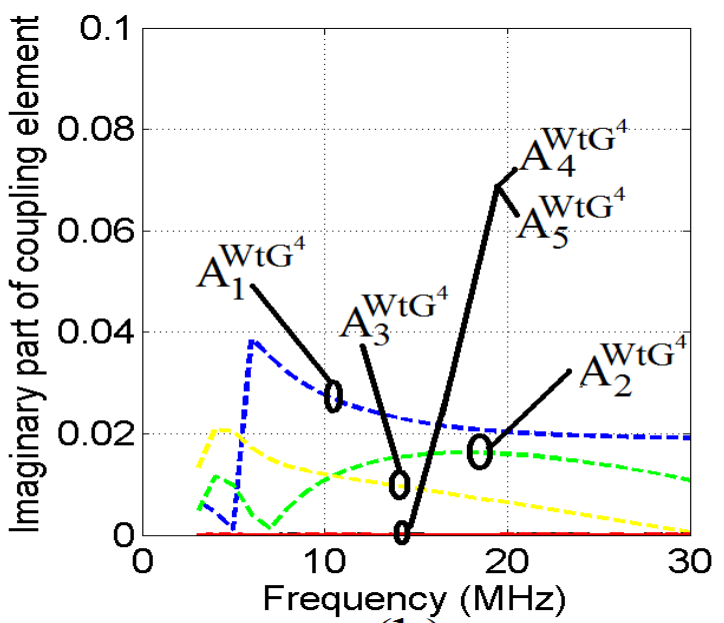

(h)

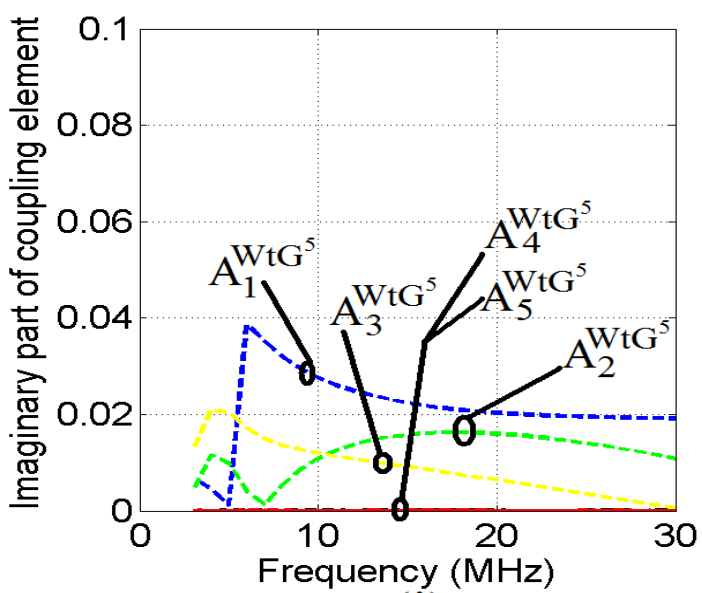

(j)

Figure 3. Spectral behavior of coupling elements of $A^{\mathrm{WtG}}$ for different $\mathrm{WtG}$ coupling schemes when the hybrid method (dashed lines) and the TEP method (solid lines) are applied in the $3-30 \mathrm{MHz}$ frequency band (the subchannel frequency spacing is equal to $0.1 \mathrm{MHz}$ ). $(a, c, e, g)$ Absolute value of real parts. $(b, d, f, h)$ Absolute value of imaginary parts. 
Nonetheless, except for this propagation analysis, a set of properties regarding the spectral efficiency of overhead HV/BPL networks, such as EMI regulations and noise, is required so that the capacity of these networks can be evaluated. In fact, TEP method exploits the already simplified and well-verified spectral efficiency properties of the hybrid method.

\section{A. EMC with other Radio services. EMI Regulations and Respective Power Constraints}

Since overhead HV/BPL networks may become both a source and a victim of EMI, a critical issue related to BPL technology has to do with the power constraints that should be imposed to ensure successful BPL coexistence with other already existing wireless and telecommunications systems [12], [15], [16], [25], [26]. Among regulatory bodies that have established regulations concerning BPL network operation and the corresponding emissions from BPL equipment, the most important are: (i) Regulations from national bodies: FCC Part 15, German Reg TP NB30, the Norwegian Proposal and the BBC/NATO Proposal [27]-[31]; and (ii) Regulations from BPL equipment manufacturers: IEEE proposal and HomePlug AV proposal [32], [33].

The comprehensive compliance testing procedures require electromagnetic field measurements at each BPL unit being part of the BPL network. As it has already presented in [9], [12], [15], [16], a simpler regulatory approach would be to avoid formal compliance tests by limiting IPSD to a level that, in most circumstances, does not produce EMI exceeding certain limits. Among the different IPSD limits proposals, the IPSD limits proposed by Ofcom for compliance with FCC Part 15 -presented in [27]-[31]- are the most cited. More specifically, for overhead HV/BPL networks, according to Ofcom, in the $3-30 \mathrm{MHz}$ frequency range maximum levels of $-60 \mathrm{dBm} / \mathrm{Hz}$ constitute appropriate IPSD limits $p(f)$ providing presumption of compliance with the current FCC Part 15 limits [9], [12], [15], [16], [31], [34], [35].

As it concerns the EMI regulations from national bodies, the electromagnetic field strength limits proposed by FCC Part 15, German Reg TP NB30, the Norwegian Proposal and the BBC/NATO Proposal are presented in [27], [29], [30]. Since Ofcom/FCC Part 15 IPSD limits are well defined, the respective IPSD limits of German Reg TP NB30, the Norwegian Proposal and the BBC/NATO Proposal can easily be determined [30].

As it concerns the IPSD limits from BPL equipment manufacturers, those of IEEE and HomePlug AV are well known and are presented in [32], [33]. However, these IPSD limits are not always EMI-harmless to other already existing telecommunications systems.

\section{B. Noise Characteristics}

According to [12], [15], [16], [20], [24], [36]-[38], two types of noise are dominant in overhead HV/BPL networks: (i) Colored background noise. It is the environmental noise depending on weather conditions, humidity, geographical location, height of cables above the ground, corona discharge, e.t.c.; and (ii) Narrowband noise. It is the result of narrowband interference from other wireless services operating at the same frequency range with overhead HV/BPL networks.

In accordance with [9], [12], as it regards the noise properties of overhead HV/BPL networks in the 3-30MHz frequency band, uniform AWGN PSD level $N(f)$ is assumed. Thoroughly examining the existing BPL noise literature [12], [15], [16], [20], [24], [34], [35], [38], these uniform AWGN/PSD levels may vary from $-95 \mathrm{dBm} / \mathrm{Hz}$ (bad 
noise scenario) to $-115 \mathrm{dBm} / \mathrm{Hz}$ (good noise scenario) with average value equal to $-105 \mathrm{dBm} / \mathrm{Hz}$ (average noise scenario). In very special cases of severe weather and aggravated EMI conditions, it is reported that AWGN/PSD levels can reach up to $-50 \mathrm{dBm} / \mathrm{Hz}$ (very bad noise scenario).

Note that as it regards the above noise characteristics of overhead HV/BPL networks, common AWGN PSD level $N(f)$ is assumed for the different modal and coupling scheme channels exploiting their significant similarities concerning BPL signal transmission. Anyway, this is a typical procedure that does not harm the generality of the following analysis [15], [20], [24], [39].

\section{Capacity}

Capacity is the maximum achievable transmission rate over a BPL channel -either modal or coupling scheme one-. It depends on the overhead HV MTL configuration, overhead HV/BPL topology, the applied coupling scheme, the imposed EMI regulations and the noise characteristics. More specifically, the capacity of an overhead HV/BPL channel is given by [9], [12], [15], [16], [40]-[42]

$$
\begin{gathered}
C \equiv C(L=K)=f_{s} \sum_{q=0}^{L-1} \log _{2}\left\{1+\left[S N R\left(3 \mathrm{MHz}+q f_{s}\right) \cdot\left|H\left(3 \mathrm{MHz}+q f_{s}\right)\right|^{2}\right]\right\} \\
S N R(f)=\langle p(f)\rangle_{L} /\langle N(f)\rangle_{L} \\
K=(30-3) \mathrm{MHz} / f_{s}
\end{gathered}
$$

where $H\{\{\}$ is the transfer function of either modal or coupling scheme channel considered, $\langle\cdot\rangle_{L}$ is an operator that converts $\mathrm{dBm} / \mathrm{Hz}$ into a linear power ratio $(\mathrm{W} / \mathrm{Hz})$, $K$ is the number of subchannels in the BPL signal frequency range of interest and $f_{s}$ is the flat-fading subchannel frequency spacing.

The cumulative capacity is defined as the cumulative upper limit of information which can reliably be transmitted over the overhead HV/BPL channel. For given frequency $f \in[3,30] \mathrm{MHz}$, overhead HVMTL configuration and coupling scheme configuration and taking into account eq. (6), cumulative capacity is determined by [40]-[42]

$$
\operatorname{CumC}(f)=C\left(L=\left\|\frac{f-3 \mathrm{MHz}}{f_{s}}\right\|+1\right)
$$

where $\|x\|$ is the nearest integer to $x$. In fact, cumulative capacity describes the aggregate capacity effect of all subchannels of the examined frequency band.

\section{Numerical Results and Discussion}

The simulation results of various types of overhead HV/BPL channels aim at highlighting to ECE students: (i) their broadband performance; (ii) how their capacity is influenced by various inherent and imposed factors; (iii) the influence of EMI regulations and noise conditions on the used techno-economic metrics; and (iv) the adaptive capacity mitigation technique that redefines the EMI regulations by taking into account noise conditions. Prior to study the previous findings, the significant convergence between hybrid and TEP method needs to be examined. 
As mentioned in Section III, since the modes supported by the overhead HV MTL configurations may be examined separately, it is assumed for simplicity that the BPL signal is injected directly into the existing EVD modes [8]-[17], [20], [34], [43]; thus, either the complicated modal analysis of [34], briefly described in Section III, is bypassed or the proposed approximations of TEP method can comfortably be applied.

For the numerical computations, the $150 \mathrm{kV}$ single-circuit overhead HV MTL configuration, depicted in Fig. 1, has been considered. In order to apply the hybrid and TEP method, according to the hybrid method, a general end-to-end BPL connection is separated into segments -network modules-, each of them comprising the successive branches encountered -see Figs. 4(a) and 4(b)-. However, the TEP method copes with overhead HV/BPL networks that comprise "LOS" transmission topologies where "LOS" topologies correspond to Line-of-Sight transmission of wireless channels (i.e., no branches are encountered). Hence, the "LOS" transmission along the average end-to-end distance $L=L_{1}+\ldots+L_{N+1}=25 \mathrm{~km}$ is assumed. Finally, with reference to Fig. 4(b), the transmitting and the receiving ends are assumed matched to the characteristic impedance of the supported modal channels [9]-[16], [18], [20], [45].

\section{A. Modal and Coupling Scheme Channels of Hybrid and TEP Method: Their Converged Transmission and Capacity Performance}

The following discussion will focus on the transmission and capacity characteristics using the techno-economic metrics of end-to-end channel attenuation and cumulative capacity associated with: (i) the CM and the DMs of the aforementioned overhead HV MTL configuration; and (ii) the WtG coupling schemes related to this overhead HV MTL configuration.

As it has already been presented in [9]-[11], to verify the convergence between hybrid and TEP method and to compare modal channels with coupling scheme ones, a representative overhead $\mathrm{HV} / \mathrm{BPL}$ topology of path length up to $25 \mathrm{~km}$ is examined, namely:

(1) The "LOS" transmission along the average end-to-end distance $L=L_{1}+\ldots+L_{N+1}=25 \mathrm{~km}$ when no branches are encountered (case A).

With respect to Fig. 4(a) and as it concerns the transmission characteristics of overhead HV/BPL channels, in Fig. 5(a), the end-to-end channel attenuation from A to B is plotted versus frequency in the $3-30 \mathrm{MHz}$ frequency band for the propagation of $\mathrm{CM}$ and $\mathrm{DM}_{i-1}, i=2, \ldots, 5$ when the $150 \mathrm{kV}$ single-circuit overhead HV MTL configuration is considered and hybrid method is applied. In Fig. 5(b), the same plots are given when the TEP method is adopted.

In Fig. 5(c), the end-to-end channel attenuation from A to B for the "LOS" transmission case A is plotted versus frequency when the hybrid method is adopted for the following coupling schemes: $\mathrm{WtG}^{1}, \mathrm{WtG}^{2}, \mathrm{WtG}^{3}, \mathrm{WtG}^{4}$ and $\mathrm{WtG}^{5}$. In Fig. 5(d), the same curves are plotted when the TEP method is applied. 
a)

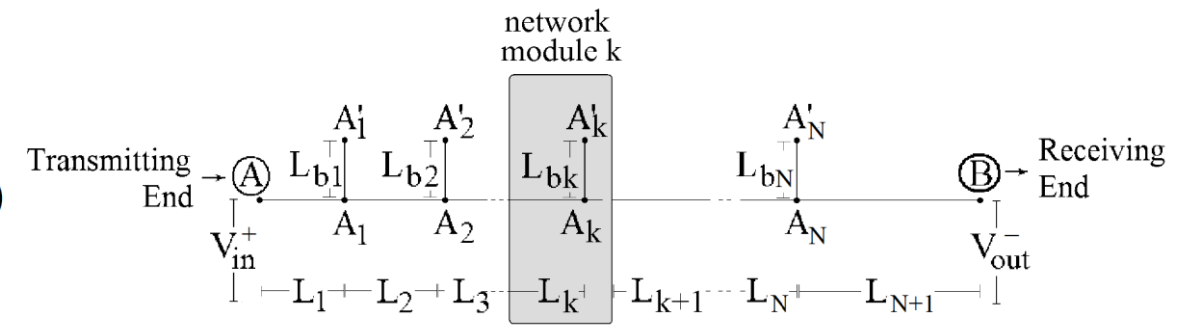

b)

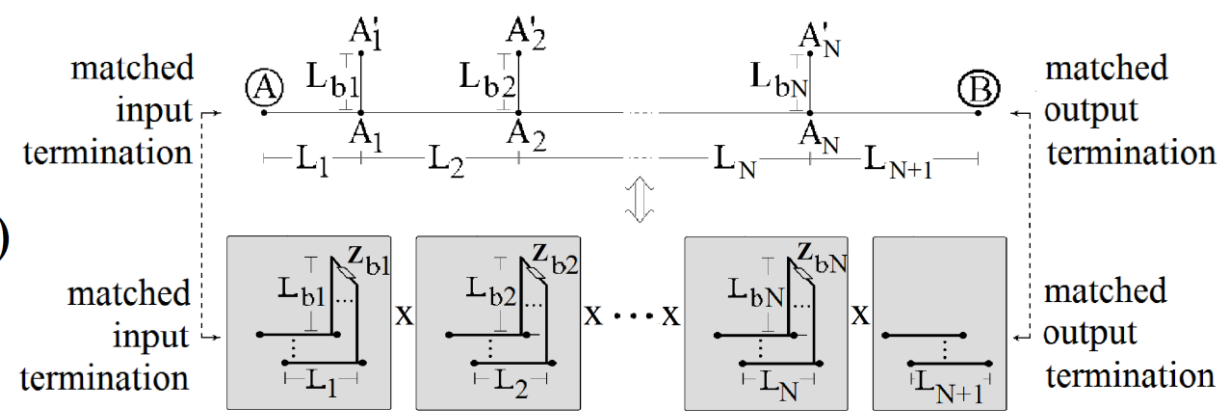

Figure 4. End-to-end BPL connection with $N$ branches. (b) In accordance with the hybrid method, an indicative overhead HV/BPL topology considered as a cascade of $N+1$ modules corresponding to $N$ branches [8]-[16].

At the same time, to investigate the capacity potential of overhead HV/BPL channels, in Fig. 6(a), the cumulative capacity of the aforementioned five modes is plotted with respect to frequency in the $3-30 \mathrm{MHz}$ frequency band when the $150 \mathrm{kV}$ single-circuit overhead HV MTL configuration, FCC Part 15 limits and average noise scenario are considered and the hybrid method is adopted. In Fig. 6(b), the same plots are given when the TEP method is applied.

In Figs. 6(c) and 6(d), similar plots are given in the case of the aforementioned indicative coupling schemes when the hybrid and TEP method is adopted, respectively.

From Figs. 5(a)-(d) and 6(a)-(d), several interesting remarks regarding transmission and capacity properties of overhead HV/BPL networks can be highlighted to ECE students:

- The simulation results of the average length "LOS" transmission channels reveal the potentially excellent communications medium of overhead HV/BPL channels -either modal or coupling scheme ones-. Already verified in [10], [12], [15], [16], [18], the entire overhead transmission power grid resembles a flat-fading transmission system with low-loss and high-capacity characteristics revealing an attractive broadband last mile alternative and SG telecommunications solution.

- As it concerns the end-to-end attenuation of modal and coupling scheme channels, TEP method achieves to provide a satisfactory approximation of their actual behavior, as verified by the hybrid method. The great success of the TEP method is that it holds this attenuation accuracy in comparison with the hybrid method results bypassing the complicated bottom-up and top-down approaches of the 


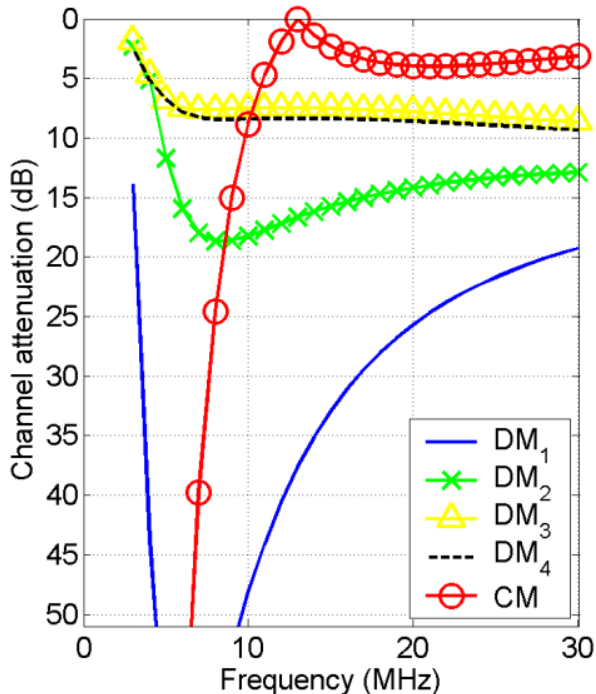

(a)

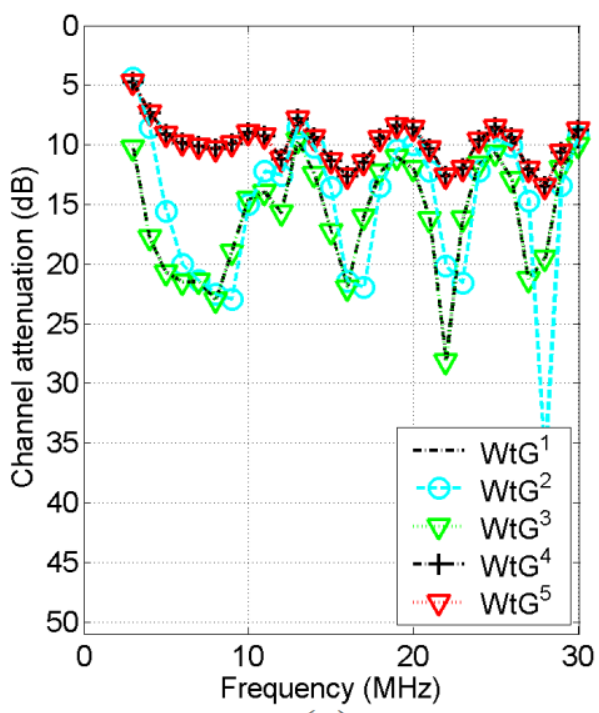

(c)

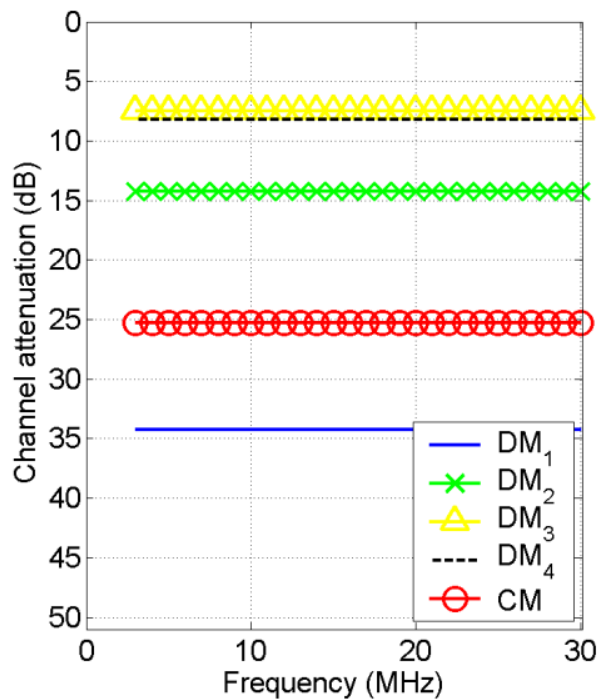

(b)

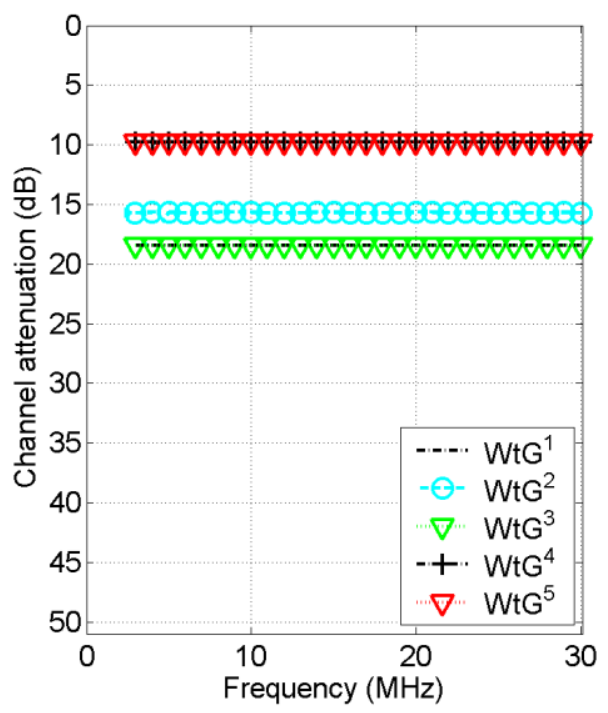

(d)

Figure 5. Channel attenuation versus frequency for the "LOS" transmission case A when the $150 \mathrm{kV}$ single-circuit overhead HV MTL configuration, FCC Part 15 limits and average noise scenario are assumed (for plot clarity reasons, the subchannel frequency spacing is equal to $1 \mathrm{MHz}$ ). (a) End-to-end attenuation of modal channels (hybrid method). (b) End-to-end attenuation of modal channels (TEP method). (c) End-to-end attenuation of WtG coupling scheme channels (hybrid method). (c) End-to-end attenuation of WtG coupling scheme channels (TEP method).

hybrid method. This is achieved by simply scaling the modal propagation constant results of Figs. 2(a)-(d) and using the constant coupling elements of Figs. 3(a)-(j). For example, the results concerning the absolute value of the modal channel attenuation presented in Fig. 5(b) come from the simple multiplication of the modal attenuation constants with 25 . Then, the results concerning the absolute 


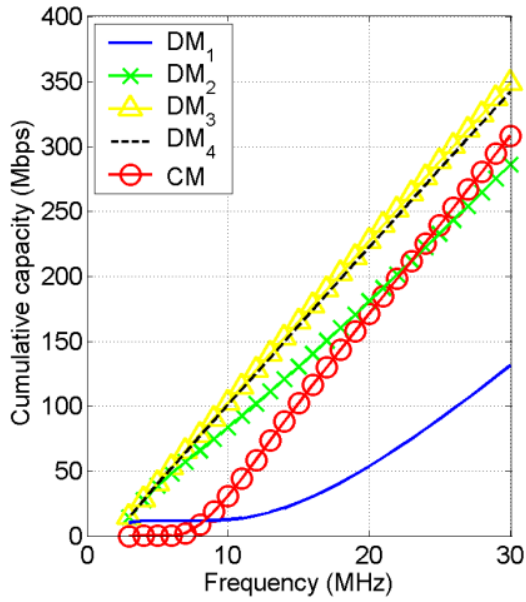

(a)

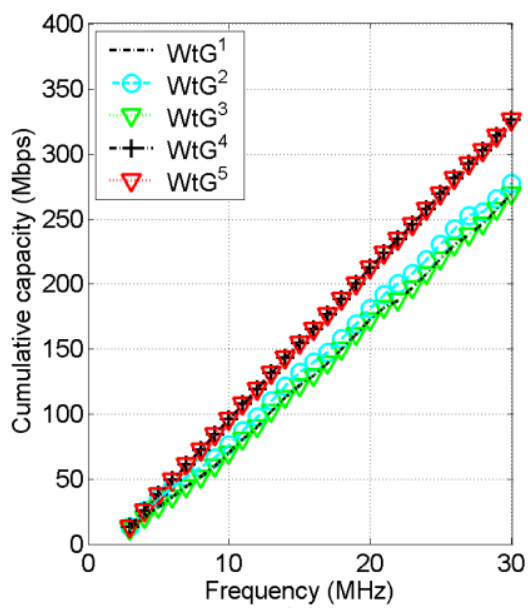

(c)

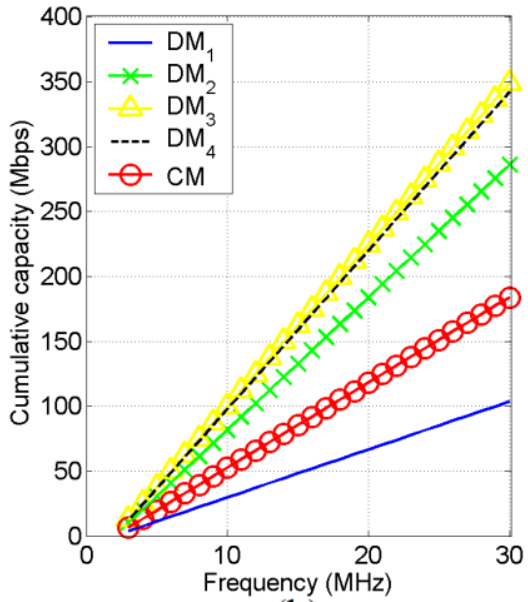

(b)

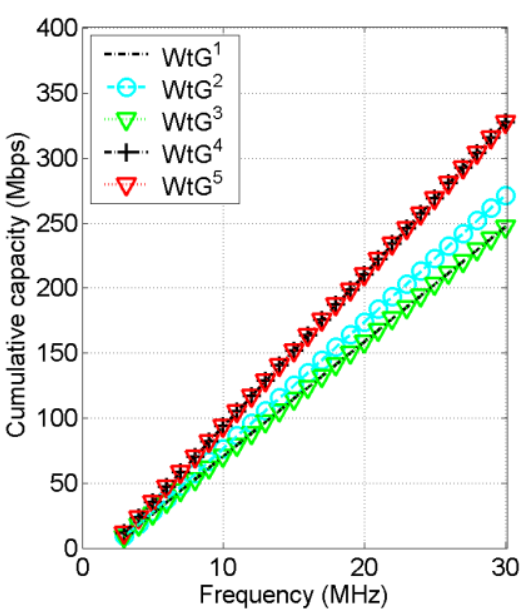

(d)

Figure 6. Cumulative capacity versus frequency for the "LOS" transmission case A when the $150 \mathrm{kV}$ single-circuit overhead HV MTL configuration, FCC Part 15 limits and average noise scenario are considered (for plot clarity reasons, the subchannel frequency spacing is equal to $1 \mathrm{MHz}$ ). (a) Cumulative capacity of modal channels (hybrid method). (b) Cumulative capacity of modal channels (TEP method). (c) Cumulative capacity of WtG coupling scheme channels (hybrid method). (d) Cumulative capacity of WtG coupling scheme channels (TEP method).

value of the channel attenuations presented in Fig. 5(d) come from the straightforward multiplication of the modal channel attenuations of Fig. 5(b) with the respective coupling elements of the applied coupling scheme. Already mentioned, note that modal propagation constants and coupling elements of TEP method are already known for given overhead HV MTL configuration and coupling scheme.

- The ECE students can observe that the spectral behavior of the end-to-end modal channel attenuation critically depends on the mode and the length of the topology examined. Already identified for the hybrid method in [10], [11], [13], [15], [18], [20], the shape and depth of the end-to-end channel attenuation of CM is primarily influenced by the penetration depth into the lossy ground and the resonance occurring inside the ground, whereas the shape and depth of the 
end-to-end channel attenuation of DMs is mainly affected by the losses and the skin-effect in the conductors.

- As it concerns the capacity of modal and coupling scheme channels, TEP method achieves to provide a very good approximation of their actual behaviour, as it is evaluated by the hybrid method. The main divergence between the capacity results of the hybrid and the TEP method is the CM modal capacity that is, anyway, vanished during the capacity evaluation of coupling scheme channels. Apart from the easy capacity computations due to the transmission and capacity assumptions of TEP method, ECE students can also observe the linear slope of cumulative capacity results that is explained by the constant terms included in eq. (6).

- Already mentioned in [9]-[11], as it concerns the transmission characteristics of overhead HV/BPL networks, WtG coupling schemes are mostly influenced by the $\mathrm{CM}$ behaviour. Here, it is validated that the same influence behaviour regarding coupling schemes and their compound modes is also observed in the case of capacity metrics. Since CM demonstrates competitive transmission and capacity results among the other supported DMs' ones, WtG coupling schemes also attain favourable results in terms of channel attenuation and cumulative capacity in comparison with other coupling schemes such as WtW ones [9], [11]. Therefore, during practical implementations in overhead HV/BPL networks, WtG coupling schemes are preferred regardless of the power grid type considered when the primary design objective is the capacity/throughput maximization.

- One of the main issues concerning the design of networks is the trade-off relations among involved system performance metrics. ECE students should understand that there are significant trade-off relations even in the design of overhead $\mathrm{HV} / \mathrm{BPL}$ networks; although WtG coupling schemes present favourable channel attenuation and capacity characteristics, the significant EMI of WtG coupling schemes to other already licensed wireless communications due to CM is the main significant drawback of WtG coupling scheme system deployment [9], [11], [29], [30]. At this point, ECE students should recognize that today's EMI regulations provide the required protection between BPL systems and other radio services regardless of the coupling scheme applied.

Without affecting the generality of the analysis, only TEP method is adopted and only one of the $\mathrm{WtG}$ coupling schemes - say $\mathrm{WtG}^{4}$ one- will be examined, hereafter.

\section{B. Influence of Overhead HV/BPL Topologies on Transmission and Capacity Metrics}

The potential transmission and capacity performance of overhead HV/BPL networks in terms of end-to-end channel attenuation and cumulative capacity, respectively, is evaluated based on the application of FCC Part 15 limits and the consideration of average noise scenario in the $3-30 \mathrm{MHz}$ frequency band when different lengths of overhead HV/BPL topologies occur.

More specifically, with reference to Fig. 4(b), similarly to the "LOS" transmission case A already presented in Section VA, other three indicative overhead HV/BPL topologies of "LOS" transmission are examined, namely:

(2) The "LOS" transmission along the average end-to-end distance $L=L_{1}+\ldots+L_{N+1}=1 \mathrm{~km}$ (case B). 
(3) The "LOS" transmission along the average end-to-end distance $L=L_{1}+\ldots+L_{N+1}=10 \mathrm{~km}($ case C).

(4) The "LOS" transmission along the average end-to-end distance $L=L_{1}+\ldots+L_{N+1}=100 \mathrm{~km}$ (case D).

As it concerns the transmission characteristics of the $150 \mathrm{kV}$ single-circuit overhead HV/BPL coupling scheme channels, in Fig. 7(a), the end-to-end channel attenuation from $\mathrm{A}$ to $\mathrm{B}$ is plotted versus frequency in the $3-30 \mathrm{MHz}$ frequency band for "LOS" transmission case $\mathrm{A}, \mathrm{B}, \mathrm{C}$ and $\mathrm{D}$ when $\mathrm{WtG}^{4}$ coupling scheme is applied. In Fig. 7(b), respective curves in terms of cumulative capacity are plotted as a function of frequency in the same frequency band for the same indicative BPL topologies when $\mathrm{WtG}^{4}$ coupling scheme is applied.

Observing Figs. 7(a) and 7(b), it is clearly demonstrated to ECE students that:

- Already verified in [9], the low-loss flat-fading transmission system with high-capacity characteristics still exist regardless of the overhead HV/BPL topology examined. Although end-to-end overhead HV connections of lengths up to $100 \mathrm{~km}$ are examined, the corresponding capacity is equal to $109 \mathrm{Mbps}$ in the 3-30MHz frequency band when FCC Part 15 limits and average noise scenario are considered. In addition, these high capacity values reveal the strong broadband potential of the entire overhead HV/BPL grid.

- ECE students should understand the critical role of end-to-end distance during the design of overhead HV/BPL networks. It can be easily pointed out that "LOS" transmission case $\mathrm{B}$ and $\mathrm{D}$ define the upper and lower channel attenuation/capacity bound, respectively, in the overhead HV MTL configuration examined. Hence, only these two overhead HV/BPL topologies will be examined, hereafter, determining the capacity limits of today's overhead HV/BPL networks when different operation scenarios are examined.

\section{Effect of EMI Regulations on the Capacity Performance of Overhead HV/BPL Network}

In this subsection, the capacity performance of overhead HV/BPL networks is investigated taking under consideration their EMI to other licensed wireless services [12], [15], [16]. Among the proposals related to the control of EMI caused by BPL operation, the six EMI regulations of Section IVA, which are characterized by their respective IPSD limits, are examined in this subsection, namely:

(i) the FCC limits proposed by FCC Part 15.

(ii) the German limits proposed by German Reg. TP NB30.

(iii) the Norwegian limits proposed by Norway.

(iv) the BBC/NATO limits proposed by BBC and NATO.

(v) the IEEE limits proposed by IEEE.

(vi) the HomePlug limits proposed by HomePlug AV.

In Fig. 8(a), the lower and upper cumulative capacity bounds of the $150 \mathrm{kV}$ single-circuit overhead HV/BPL channels have been plotted in the $3-30 \mathrm{MHz}$ frequency band in order to examine the effect of the FCC limits when $\mathrm{WtG}^{4}$ coupling scheme is applied and average noise scenario is assumed. In Figs. 8(b)-(f), similar curves are given in the case of German, Norwegian, BBC/NATO, IEEE and HomePlug limits, respectively. 


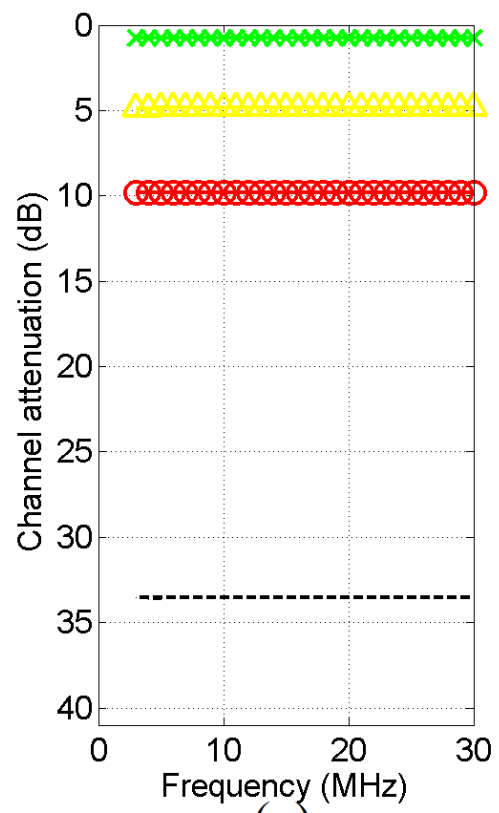

(a)

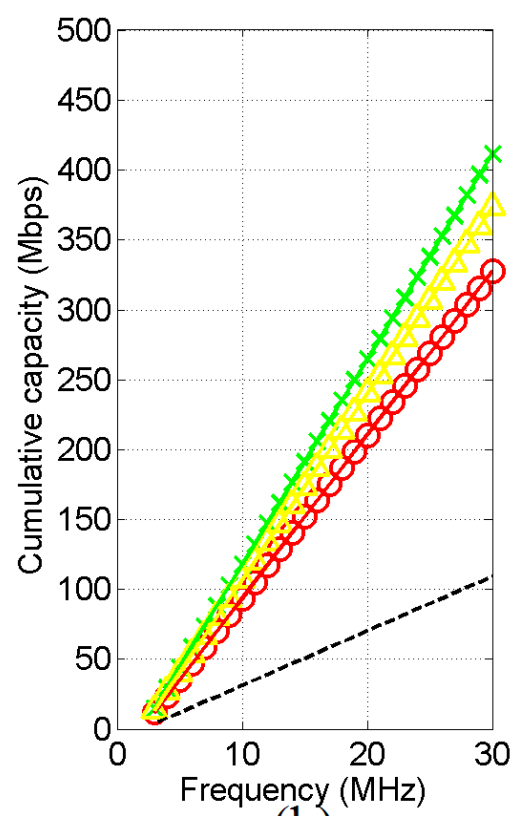

(b)

Figure 7. Transmission and capacity metrics of the $150 \mathrm{kV}$ single-circuit overhead HV MTL configuration versus frequency for "LOS" transmission case A $(\theta)$, case $B(*)$, case $C(\stackrel{\Delta}{*})$ and case D (ש- ) when FCC Part 15 limits and average noise scenario are considered and $\mathrm{WtG}^{4}$ coupling scheme is applied (for plot clarity reasons, the subchannel frequency spacing is equal to $1 \mathrm{MHz}$ ). (a) End-to-end channel attenuation. (b) Cumulative capacity.

From Figs. 8(a)-(f), several important capacity issues can be highlighted:

- ECE students can identify that the choice of EMI regulations remains a crucial decision regarding future's overhead HV/BPL broadband expansion. The tighter versions of EMI regulations combined with the very long connections push overhead HV/BPL networks to their broadband extinction; under these EMI regulations, overhead HV/BPL networks do not exhibit any capacity advantage in comparison with the vintage SCADA networks.

- In addition, ECE students can identify capacity differences of the order of hundreds of Mbps among different EMI regulations. This fact reveals the need of a slight relaxation for such long-range systems so that corresponding relaxed IPSD limits will lead to a considerable capacity increase. This adjustment may be accompanied with the use of appropriate adaptive mitigation techniques such as the adoption of different EMI regulations depending on telecommunications EMC needs, the imposition of power masking adaptive to local traffic, multi-hop transmission and cooperative communications [12], [15], [16].

- ECE students should also recognize the importance of cooperative communications. The promotion of standardized topologies concept among HV/BPL, MV/BPL and LV/BPL networks may help towards a centralized cooperative BPL network structure. This is a good reason for unveiling the need for intraoperability/interoperability of overhead HV/BPL networks with other SG broadband technologies (see also Section VE). 


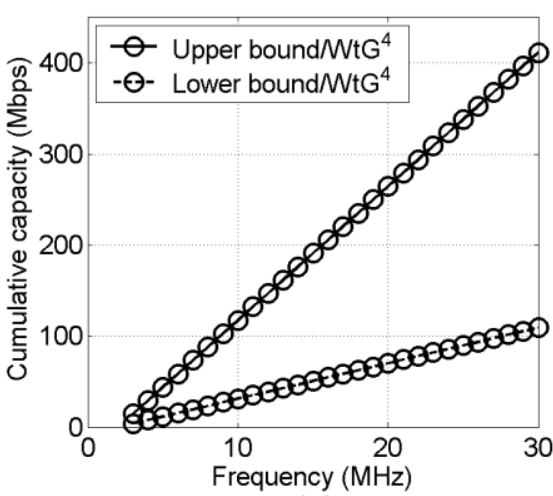

(a)

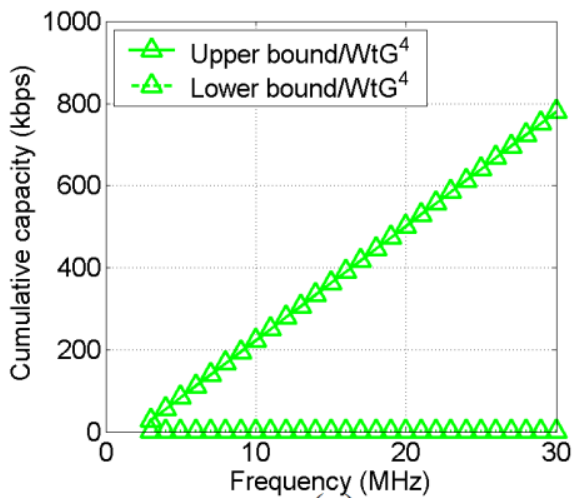

(c)

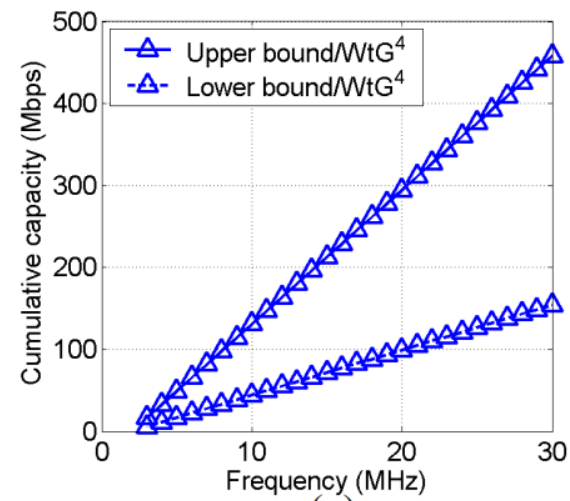

(e)

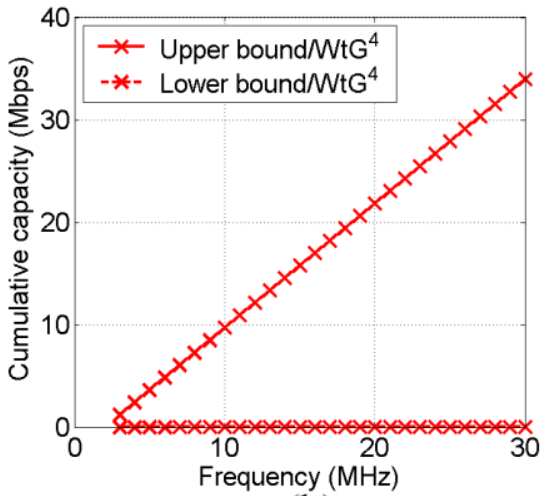

(b)

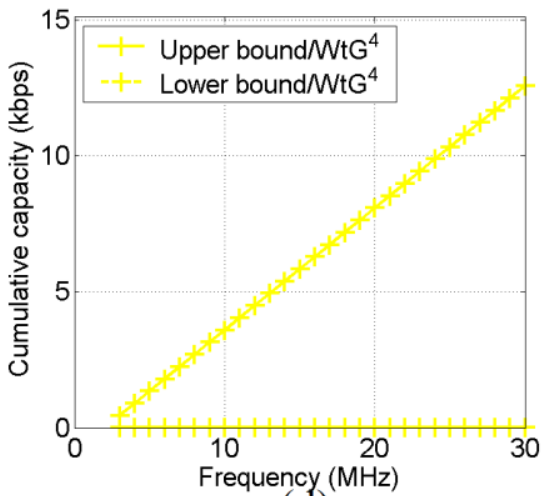

(d)

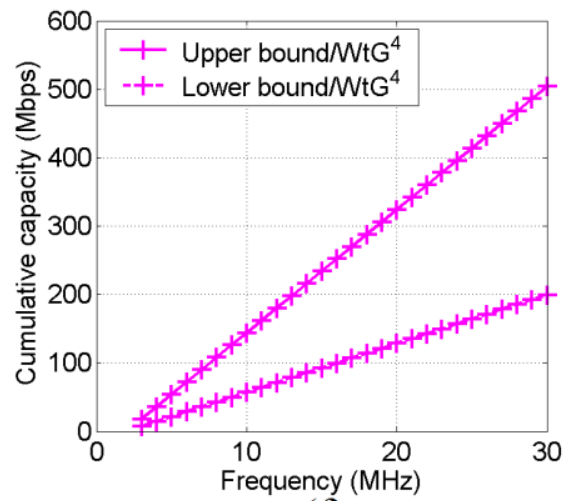

(f)

Figure 8. Upper and lower cumulative capacity bounds of the $150 \mathrm{kV}$ single-circuit overhead HV MTL configuration versus frequency when $\mathrm{WtG}^{4}$ coupling scheme is applied for different EMI regulations (for plot clarity reasons, the subchannel frequency spacing is equal to $1 \mathrm{MHz}$ ). (a) FCC limits. (b) German limits. (c) Norwegian limits. (d) BBC/NATO limits. (e) IEEE limits. (f) HomePlug limits.

- When relaxed EMI regulations are adopted -such as FCC, IEEE and HomePlug limits-, the occurred lower capacity bounds unveil the dynamic broadband SG character of overhead HV/BPL networks. The fact that an extended portfolio of SG applications needs no more than $15-20 \mathrm{Mbps}$ combined with the findings of lower capacity bounds render overhead HV/BPL lines as a very efficient SG telecommunications platform. 
D. The Combined Effect of EMI Regulations and Noise Conditions on Capacity Performance of Overhead HV/BPL Networks

Apart from the EMI regulations and the de-facto long-range nature of overhead HV/BPL topologies, in order to design high-bitrate overhead HV/BPL networks with capacities in the range of Gbps, the detailed knowledge of the noise environments is imperative [8]-[11].

Opposite to many other broadband telecommunications systems, such as optical fiber networks, and as it has already been reported in Section IVB, overhead HV/BPL noise presents high variability. This is due to the fact that overhead HV/BPL noise depends either on inherent environmental factors or on imposed narrowband interference conditions [9], [15], [16], [20], [28], [46], [47]. Actually, the significant differences in $\mathrm{dBm}$ among different noise levels are reflected on respective differences in the capacity of overhead HV/BPL networks.

In fact, the combined effect of different EMI regulations with peculiar noise conditions creates important capacity fluctuations during the operation of overhead HV/BPL networks. Depending on the capacity thresholds that are imposed by various SG applications and EMI requirements, a more general EMI regulation framework may be introduced; the IPSD limits can be adaptively tuned in local or temporal basis in order to mitigate capacity losses caused by either inherent or imposed factors across overhead HV/BPL networks bypassing universal fixed strict EMI regulations.

The following discussion focuses on the spectral efficient capabilities in terms of capacity in the $3-30 \mathrm{MHz}$ frequency band through the application of the six EMI regulations of Section IVA when different noise environments that range from the very bad noise scenario to the good one occur. Based on the "LOS" overhead HV/BPL topologies of $1 \mathrm{~km}$ (case B) and 100km (case D) that define the upper and lower capacity bounds, respectively, the following analysis tries to investigate: (i) the influence of different noise conditions to overall capacity; (ii) the combined impact of noise and EMI regulations on the broadband capacity performance of overhead HV/BPL networks; and (iii) the mitigation of capacity losses due to noise variability through the adoption of more relaxed EMI regulations.

In Fig. 9(a), the lower and upper capacity bounds of the $150 \mathrm{kV}$ single-circuit overhead HV/BPL channels in the $3-30 \mathrm{MHz}$ frequency band have been plotted versus uniform AWGN/PSD levels when $\mathrm{WtG}^{4}$ coupling scheme and FCC limits are considered. The uniform AWGN/PSD levels range from $-50 \mathrm{dBm} / \mathrm{Hz}$ (very bad noise scenario) to $-115 \mathrm{dBm} / \mathrm{Hz}$ (good noise scenario). In Figs. 9(b)-(f), similar curves are given in the case of German, Norwegian, BBC/NATO, IEEE and HomePlug limits, respectively.

Observing Figs. 9(a)-(f), ECE students can deduce certain interesting conclusions:

- It is revealed how significant for BPL transmission is the noise variance and EMI regulations as well as their interaction. In fact, capacity differences of the order of hundreds of Mbps in the 3-30MHz frequency band are observed among different combinations of AWGN/PSD and IPSD levels; say, in the case of FCC limits, capacity differences up to approximately $500 \mathrm{Mbps}$ are observed between the best and the worst BPL transmission cases. 


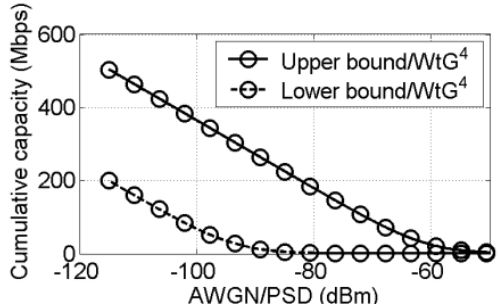

(a)

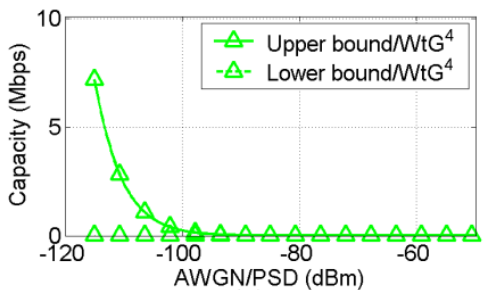

(c)

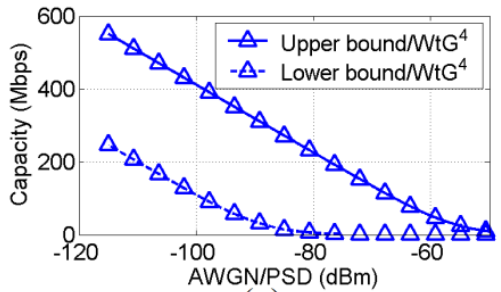

(e)

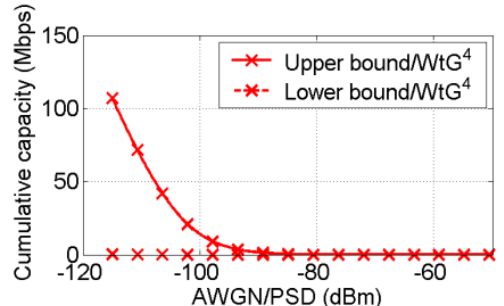

(b)

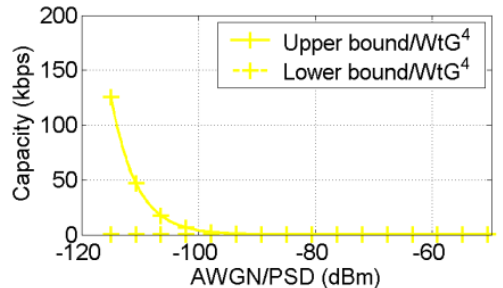

(d)

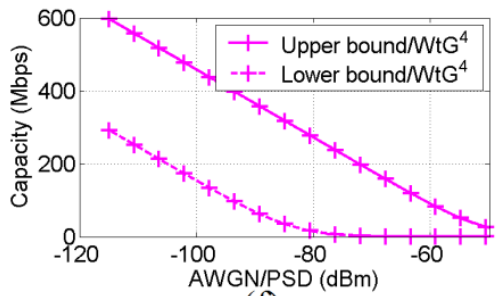

(f)

Figure 9. Upper and lower capacity bounds of the 150kV single-circuit overhead HV MTL configuration versus uniform AWGN/PSD when $\mathrm{WtG}^{4}$ coupling scheme is applied for different EMI limits (the subchannel frequency spacing is equal to $1 \mathrm{MHz}$ ). (a) FCC limits. (b) German limits. (c) Norwegian limits. (d) BBC/NATO limits. (e) IEEE limits. (f) HomePlug limits.

- Compared to other relevant capacity results of overhead and underground LV/BPL and MV/BPL networks [12], [15], [16], if good and average noise conditions are combined with appropriate EMI regulations, overhead HV/BPL networks present notable capacity performance permitting their operation as backbone networks. Actually, during these optimum operation conditions, overhead HV/BPL networks can concentrate local traffic from other already existing surrounding broadband networks if appropriate topologies are employed. However, before overhead HV/BPL systems interoperate with other broadband technologies -wireline, such as fiber and DSL, and wireless, such as WiFi and WiMax-, the overhead HV/BPL networks need to intraoperate with other surrounding overhead and underground MV/BPL and LV/BPL networks exploiting the scalable capacity principles [8]-[16].

- If 100km-long "LOS" transmission topologies are adopted, capacity performance of overhead HV/BPL networks is seriously damaged even if power injection is defined by the highest today's IPSD limits that respect EMC regulations -i.e., FCC limits-. Numerically, when very bad noise scenario occurs and FCC limits are applied, the maximum capacity of overhead HV/BPL networks does not exceed 3.3Mbps. Therefore, the harsh noise environments that derive from either environmental factors or narrowband interference conditions pulverize overhead 
HV/BPL network broadband perspective [9], [15], [16], [20], [28], [46]-[48]. Similarly to the numerical results of Section VC when severe noise conditions occur, overhead HV/BPL networks do not exhibit any capacity advantage in comparison with the SCADA networks.

\section{E. Converting Disadvantage into Advantage: The Mitigation of Capacity Losses due to Noise Conditions Using Adaptive EMI Regulations}

In Section VD, it has been clearly shown that if severe noise conditions concur with strict EMI regulations, this combination annihilates broadband capacity potential of overhead HV/BPL networks. The ECE students should understand that, reversing the arrow of IPSD limits through the appropriate adjustment of EMI regulations via their respective IPSD limits, the capacity effect of severe noise conditions can be mitigated ensuring scalable capacities among already installed overhead HV/BPL networks that are characterized by different noise conditions.

In this subsection, the adaptive adjustment of IPSD limits that depends on the weather and various EMC conditions is proposed. This capacity mitigation technique primarily ensures scalable capacities among overhead HV/BPL networks and secondarily guarantees the required symbiosis of overhead HV/BPL networks with other SG telecommunications systems. Given a capacity threshold, appropriate IPSD limits are determined so as to counterbalance capacity losses due to different AWGN/PSDs when different overhead HV/BPL topologies occur. More specifically, in order to ensure that upper and lower capacity bounds are perfectly adjusted to the required capacity threshold, appropriate upper and lower IPSD limits are determined, respectively.

In Fig. 10(a), these upper and lower IPSD limits of the $150 \mathrm{kV}$ single-circuit overhead HV MTL configuration in the $3-30 \mathrm{MHz}$ frequency band have been plotted versus uniform AWGN/PSD levels when $\mathrm{WtG}^{4}$ coupling scheme is applied and the capacity threshold is assumed equal to 100Mbps. The uniform AWGN/PSD levels range from $-95 \mathrm{dBm} / \mathrm{Hz}$ (bad noise scenario) to $-115 \mathrm{dBm} / \mathrm{Hz}$ (good noise scenario). In Figs. 10(b)-(d), similar curves are given when the capacity threshold is assumed equal to $200 \mathrm{Mbps}$, 300Mbps and 400Mbps, respectively.

Observing Figs. 10(a)-(d), the following remarks can be highlighted to ECE students:

- The schematically linear behavior of IPSD limits in respect with AWGN/PSD levels for given capacity threshold, which is observed in the previous figures, is mathematically justified by studying eqs (6) and (7); in order to maintain a fixed capacity threshold, as it is described in eq. (6), a fixed ratio between IPSD limits and AWGN/PSD levels is required. This fixed ratio, which is given in eq. (7), further entails a linear dependence relation in $\mathrm{dB}$ between IPSD limits and 


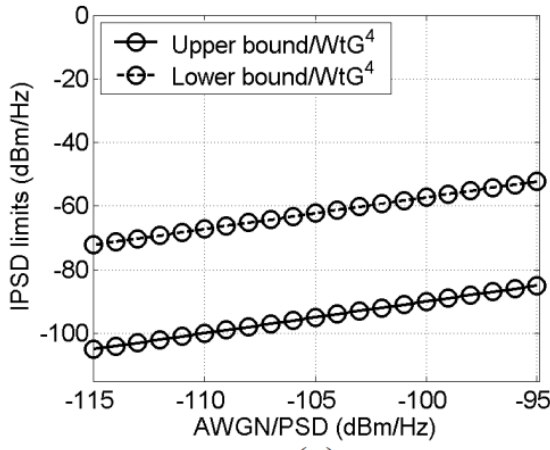

(a)

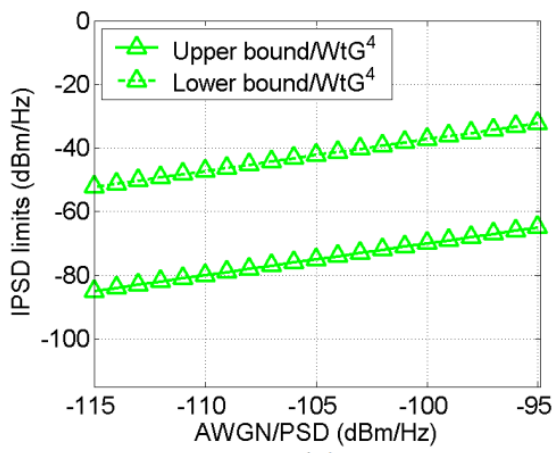

(c)

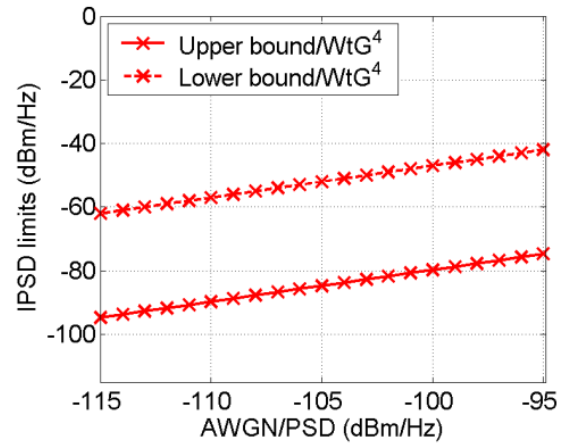

(b)

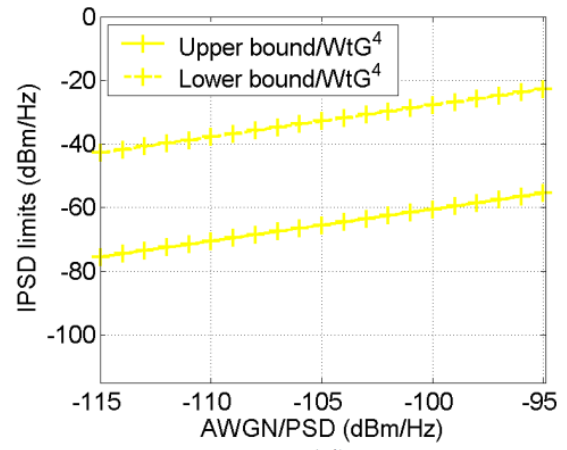

(d)

Figure 10. Upper and lower IPSD limits of the $150 \mathrm{kV}$ single-circuit overhead HV MTL configuration versus uniform AWGN/PSD when $\mathrm{WtG}^{4}$ coupling scheme is applied for different capacity thresholds (the subchannel frequency spacing is equal to $1 \mathrm{MHz}$ ). (a) Capacity threshold of $100 \mathrm{Mbps}$. (b) Capacity threshold of $200 \mathrm{Mbps}$. (c) Capacity threshold of $300 \mathrm{Mbps}$. (d) Capacity threshold of $400 \mathrm{Mbps}$.

AWGN/PSD levels. Therefore, capacity losses or gains due to weather and EMI conditions can be efficiently regulated by appropriately raising or decreasing IPSD limits, respectively. The level of IPSD limits adjustment can be accurately estimated using either the mathematical analysis based on Section IVC or schematic rules of thumb such as those of Figs. 10(a)-(d).

- It is obvious that the lower capacity bounds of overhead HV MTL configurations require higher IPSD limits in comparison with the upper capacity bounds so that a common capacity threshold among different overhead HV/BPL topologies can be ensured. Moreover, as the capacity threshold raises and/or noise conditions deteriorate, so do the respective upper and lower IPSD limits.

- By appropriately adjusting IPSD limits, a great number of capacity thresholds can be set regardless of the considered overhead HV MTL configuration and the noise environment. Hence, the concept of scalable capacity among overhead HV/BPL networks is comfortably ensured through this adaptive capacity mitigation technique. Anyway, this capacity countermeasure, which defines a strong technoeconomic design tool, permits the further coexistence among overhead HV/BPL networks of different overhead HV/BPL topologies.

- Despite the fact that every capacity threshold can theoretically be achieved across a transmission BPL network by simply tuning IPSD limits across its compound overhead HV/BPL networks, this common capacity goal cannot be practically 
achieved in all the cases due to various EMC requirements. Except for the IPSD limit restrictions concerning human exposure to radio frequency electromagnetic fields [49], [50], other already licensed telecommunications services that surround overhead HV/BPL networks and operate at the same frequency range with overhead HV/BPL systems, demand their EMI protection.

- Nevertheless, the EMI protection of the communications systems that surround overhead HV/BPL networks can be fully achieved using suitable IPSD limits. These IPSD limits may be significantly more relaxed than those of today's EMI regulations. Since overhead HV/BPL networks are mainly located in remote or distant areas, the requirements for EMI protection in these zones remain very low due to the scarcity of close human and intense communications activities. Hence, there is the flexibility of allowing higher ad-hoc IPSD limits when there are no surrounding potential EMI victims. Consequently, depending on spatial and temporal EMI criteria, a more flexible EMI regulation approach, such as this of Figs. 10(a)-(d), is feasible taking under consideration only the ad-hoc EMI limits instead of using the universal fixed EMI regulations that are dramatically pessimistic in broadband performance terms.

- From different courses of their ECE program, ECE students have the opportunity to learn about MIMO technology advances. Recently, the concept of multiconductor diversity via introduction of MIMO/BPL technology makes its first but very promising and robust steps [39]-[43]. Overhead MIMO/HV/BPL technology permits a boost of the overall capacity, link reliability, diversity and range without additional bandwidth or transmit power when high IPSD regulations are adopted. New overhead MIMO/HV/BPL networks will permit a wide variety of more sophisticated SG applications such as real-time video surveillance/monitoring, diagnostics, distribution automation applications and real broadband internet connections to local surrounding communities to be introduced.

\section{Conclusions}

This paper presents TEP method that is suitable for the study and the design of overhead HV/BPL networks from ECE students. In addition, TEP method demonstrates to undergraduate ECE students either the interaction between Microwave Engineering and Engineering Economics or the broadband potential of overhead HV/BPL networks when different overhead HV/BPL topologies, EMI regulations and noise conditions occur.

Based on the numerical results of techno-economic metrics such as end-to-end channel attenuation and capacity, major features of overhead HV/BPL networks have been reviewed for use in future transmission power grid networks and SG. In the light of information theory, the significant capacity performance of all considered overhead HV/BPL topologies is revealed. This broadband potential can further be enhanced by exploiting adaptive EMI regulations, the concept of scalable capacities, standardized topologies among different overhead and underground HV/BPL, MV/BPL and LV/BPL networks and topologies, wiser trade-offs among IPSD limits, noise, EMI protection and capacity thresholds, multi-hop BPL transmission, cooperative communications, and the integration of MIMO technology.

Especially, through the adoption of adaptive EMI regulations, which can be tuned according to noise conditions and other spatial and temporal EMI criteria, capacity 
differences among different overhead HV/BPL topologies and noise conditions can efficiently be mitigated. This adaptive capacity mitigation technique offers significant results concerning the topic of scalable capacities among different overhead HV/BPL networks and sets an important step towards the design/operation of faster, more interoperable/intraoperable and more electromagnetic compatible BPL networks in the oncoming SG.

\section{CONFLICTS OF INTEREST}

The author declares that there is no conflict of interests regarding the publication of this paper.

\section{REFERENCES}

[1] R. E. Collin, Foundations for microwave engineering, John Wiley \& Sons, 2007.

[2] D. M. Pozar, Microwave Engineering, Reading, MA, USA: Addison-Wesley Publishing Company, 1990.

[3] L. T. Blank, A. J., Tarquin, and S. Iverson, Engineering Economy, New York, USA: McGraw-Hill, 2005.

[4] G. E. Chatzarakis, S. N. Livieratos, and G. N. Miliaras, "An Integrated Method for Studying the Telegrapher's and Klein-Gordon Equations," International Journal of Mathematical Education, vol. 2, no. 2, pp. 83-98, 2012.

[5] G. E. Chatzarakis, M. D. Tortoreli, and P. G. Cottis, "Teaching to undergraduates the optimum power transfer to a load under constraints," IJEEE (International Journal of Electrical Engineering Education), vol. 41, no. 2, pp. 126-136, 2004. DOI: $10.7227 /$ ijeee.41.2.4

[6] R. Stoecker, "Evaluating and rethinking the case study," The sociological review, vol. 39, no.1, pp. 88-112, 1991. DOI: 10.1111/j.1467-954X.1991.tb02970.x

[7] G. E. Chatzarakis, "Nodal Analysis Optimization Based on the Use of Virtual Current Sources: A Powerful New Pedagogical Method," IEEE Transactions on Education, vol. 52, no. 1, pp. 144-150, Feb. 2009. DOI: 10.1109/te.2008.921459

[8] A. G. Lazaropoulos, "Review and progress towards the common broadband management of high-voltage transmission grids: model expansion and comparative modal analysis," ISRN Electronics, vol. 2012, Article ID 935286, pp. 1-18, 2012. DOI: $10.5402 / 2012 / 935286$

[9] A. G. Lazaropoulos, "Deployment concepts for overhead high voltage broadband over power lines connections with two-hop repeater system: Capacity countermeasures against aggravated topologies and high noise environments," Progress in Electromagnetics Research B, vol. 44, pp. 283-307, 2012. DOI: 10.2528/PIERB12081104

[10] A. G. Lazaropoulos, "Broadband transmission characteristics of overhead highvoltage power line communication channels," Progress in Electromagnetics Research B, vol. 36, pp. 373-398, 2012. DOI: 10.2528/PIERB11091408

[11] A. G. Lazaropoulos, "Broadband transmission and statistical performance properties of overhead high-voltage transmission networks," Journal of Computer Networks and Commun., 2012, article ID 875632, 2012. DOI: $10.1155 / 2012 / 875632$ 
[12] A. G. Lazaropoulos, "Review and Progress towards the Capacity Boost of Overhead and Underground Medium-Voltage and Low-Voltage Broadband over Power Lines Networks: Cooperative Communications through Two- and ThreeHop Repeater Systems," ISRN Electronics, vol. 2013, Article ID 472190, pp. 1-19, 2013. DOI: $10.1155 / 2013 / 472190$

[13] A. G. Lazaropoulos and P. G. Cottis, "Transmission characteristics of overhead medium voltage power line communication channels," IEEE Trans. Power Del., vol. 24, no. 3, pp. 1164-1173, Jul. 2009. DOI: 10.1109/tpwrd.2008.2008467

[14] A. G. Lazaropoulos and P. G. Cottis, "Broadband transmission via underground medium-voltage power lines-Part I: transmission characteristics," IEEE Trans. Power Del., vol. 25, no. 4, pp. 2414-2424, Oct. 2010. DOI: 10.1109/tpwrd.2010.2048929

[15] A. G. Lazaropoulos and P. G. Cottis, "Capacity of overhead medium voltage power line communication channels," IEEE Trans. Power Del., vol. 25, no. 2, pp. 723-733, Apr. 2010. DOI: 10.1109/tpwrd.2009.2034907

[16] A. G. Lazaropoulos and P. G. Cottis, "Broadband transmission via underground medium-voltage power lines-Part II: capacity," IEEE Trans. Power Del., vol. 25, no. 4, pp. 2425-2434, Oct. 2010. DOI: 10.1109/tpwrd.2010.2052113

[17] T. Calliacoudas and F. Issa, "Multiconductor transmission lines and cables solver," An efficient simulation tool for plc channel networks development," presented at the IEEE Int. Conf. Power Line Communications and Its Applications, Athens, Greece, Mar. 2002.

[18] N. Suljanović, A. Mujčić, M. Zajc, and J. F. Tasič, “Approximate computation of high-frequency characteristics for power line with horizontal disposition and middle-phase to ground coupling," Electric Power Systems Research, 69(1), 1724. DOI: 10.1016/j.epsr.2003.07.005

[19] M. Zajc, N. Suljanović, A. Mujčić, and J. F. Tasič, "Frequency characteristics measurement of overhead high-voltage power-line in low radio-frequency range," IEEE Trans. Power Del., vol. 22, no. 4, pp. 2142-2149, Oct. 2007. DOI: 10.1109/tpwrd.2007.905369

[20] P. Amirshahi and M. Kavehrad, "High-frequency characteristics of overhead multiconductor power lines for broadband communications," IEEE J. Sel. Areas Commun., vol. 24, no. 7, pp. 1292-1303, Jul. 2006. DOI: $10.1109 /$ jsac.2006.874399

[21] M. D'Amore and M. S. Sarto, "A new formulation of lossy ground return parameters for transient analysis of multiconductor dissipative lines," IEEE Trans. Power Del., vol. 12, no. 1, pp. 303-314, Jan. 1997. DOI: 10.1109/61.568254

[22] M. D'Amore and M. S. Sarto, "Simulation models of a dissipative transmission line above a lossy ground for a wide-frequency range-Part I: Single conductor configuration," IEEE Trans. Electromagn. Compat., vol. 38, no. 2, pp. 127-138, May 1996. DOI: 10.1109/15.494615

[23] M. D'Amore and M. S. Sarto, "Simulation models of a dissipative transmission line above a lossy ground for a wide-frequency range-Part II: Multi-conductor configuration," IEEE Trans. Electromagn. Compat., vol. 38, no. 2, pp. 139-149, May 1996. DOI: $10.1109 / 15.494616$

[24] P. Amirshahi, "Broadband access and home networking through powerline networks," Ph.D. dissertation, Pennsylvania State Univ., University Park, PA, May 2006. 
[25] S. Galli and O. Logvinov, "Recent developments in the standardization of power line communications within the IEEE," IEEE Commun. Mag., vol. 46, no. 7, pp. 64-71, Jul. 2008. DOI: 10.1109/mcom.2008.4557044

[26] D. Fenton and P. Brown, "Some aspects of benchmarking high frequency radiated emissions from wireline communications systems in the near and far fields," in Proc. IEEE Int. Symp. on Power Line Communications and its Applications, Malmö, Sweden, Apr. 2001, pp. 161-167.

[27] M. Gebhardt, F. Weinmann, and K. Dostert, "Physical and regulatory constraints for communication over the power supply grid," IEEE Commun. Mag., vol. 41, no. 5, pp. 84-90, May 2003. DOI: 10.1109/mcom.2003.1200106

[28] Ofcom, "Amperion PLT Measurements in Crieff," Ofcom, Tech. Rep., Sept. 2005.

[29] NTIA, "Potential interference from broadband over power line (BPL) systems to federal government radio communications at 1.7-80 MHz Phase 1 Study Vol. 1," NTIA Rep. 04-413, Apr. 2004.

[30] NATO, "HF Interference, Procedures and Tools (Interférences HF, procédures et outils) Final Report of NATO RTO Information Systems Technology," RTO-TRISTR-050, Jun. 2007.

[31] FCC, "In the Matter of Amendment of Part 15 regarding new requirements and measurement guidelines for Access Broadband over Power Line Systems," FCC 04-245 Report and Order, Jul. 2008.

[32] IEEE 1901 Working Group, "IEEE Standard for Broadband over Power Line Networks: Medium Access Control and Physical Layer Specifications," Tech. Report, 2010.

[33] K. Youge, "HomePlug AV Technical Overview," in Proc. IEEE Int. Symp. Power Line Communications and Its Applications, Orlando, Florida, USA, Mar. 2006.

[34] Ofcom, "DS2 PLT Measurements in Crieff," Ofcom, Tech. Rep. 793 (Part 2), May 2005.

[35] Ofcom, “Ascom PLT Measurements in Winchester," Ofcom, Tech. Rep. 793 (Part 1), May 2005.

[36] M. Götz, M. Rapp, and K. Dostert, "Power line channel characteristics and their effect on communication system design," IEEE Commun. Mag., vol. 42, no. 4, pp. 78-86, Apr. 2004. DOI: 10.1109/mcom.2004.1284933

[37] R. Aquilué, I. Gutierrez, J. L. Pijoan, and G. Sánchez, "High-voltage multicarrier spread-spectrum system field test," IEEE Trans. Power Del., vol. 24, no. 3, pp. 1112-1121, Jul. 2009. DOI: 10.1109/tpwrd.2008.2002847

[38] National Energy Technology Laboratory, "HV-BPL Phase 2, Field Test Report", Tech. Report, 2009.

[39] T. Sartenaer and P. Delogne, "Deterministic modelling of the (Shielded) outdoor powerline channel based on the multiconductor transmission line equations," IEEE J. Sel. Areas Commun., vol. 24, no. 7, pp. 1277-1291, Jul. 2006. DOI: $10.1109 /$ jsac.2006.874423

[40] A. G. Lazaropoulos, “Green overhead and underground Multiple-Input MultipleOutput medium voltage broadband over power lines networks: Energy-efficient power control," Journal of Global Optimization, 57(3), 997-1024, 2013. DOI: 10.1007/s10898-012-9988-y.

[41] A. G. Lazaropoulos, "Overhead and underground MIMO low voltage broadband over power lines networks and EMI regulations: Towards greener capacity 
performances." Computers \& Electrical Engineering, 39(7), 2214-2230, 2013. DOI: 10.1016/j.compeleceng.2013.02.003

[42] A. G. Lazaropoulos, "Broadband over Power Lines (BPL) Systems Convergence: Multiple-Input Multiple-Output (MIMO) Communications Analysis of Overhead and Underground Low-Voltage and Medium-Voltage BPL Networks," ISRN Power Engineering, vol. 2013, 30, 2013. DOI: 10.1155/2013/517940

[43] T. Sartenaer, "Multiuser communications over frequency selective wired channels and applications to the powerline access network" Ph.D. dissertation, Univ. Catholique Louvain, Louvain-la-Neuve, Belgium, Sep. 2004.

[44] C. R. Paul, Analysis of Multiconductor Transmission Lines. New York: Wiley, 1994.

[45] N. Suljanović, A. Mujčić, M. Zajc, and J. F. Tasič, "High-frequency characteristics of high-voltage power line," in Proc. IEEE Int. Conf. on Computer as a Tool, Ljubljana, Slovenia, Sep. 2003, pp. 310-314.

[46] M. Zimmermann and K. Dostert, "Analysis and modeling of impulsive noise in broad-band powerline communications," IEEE Trans. Electromagn. Compat., vol. 44, no. 1, pp. 249-258, Feb. 2002. DOI: 10.1109/15.990732

[47] M. Katayama, T. Yamazato, and H. Okada, "A mathematical model of noise in narrowband power line communication systems," IEEE J. Sel. Areas Commun., vol. 24, no. 7, pp. 1267-1276, Jul. 2006. DOI: 10.1109/jsac.2006.874408

[48] P. Amirshahi and M. Kavehrad, "Medium voltage overhead powerline broadband communications; Transmission capacity and electromagnetic interference," in Proc. IEEE Int. Symp. Power Line Commun. Appl., Vancouver, BC, Canada, Apr. 2005, pp. 2-6.

[49] IEEE - International Committee on Electromagnetic Safety ICES, "Standard for safety levels with respect to human exposure to radio frequency electromagnetic fields, $3 \mathrm{kHz}$ to $300 \mathrm{GHz}$," IEEE Std. C95.1-1991, 1992.

[50] International Commission on Non-Ionizing Radiation Protection, "Guidelines for limiting exposure to time-varying electric, magnetic, and electromagnetic fields (up to 300 GHz)," Health Phys., vol. 74, no. 4, pp. 494-522, Apr. 1998.

Article copyright: $(\mathbb{C} 2015$ Athanasios G. Lazaropoulos. This is an open access article distributed under the terms of the Creative Commons Attribution 4.0 International License, which permits unrestricted use and distribution provided the original author and source are credited. 